\title{
Towards self-consistent plasma modelisation in presence of Neoclassical Tearing Mode and sawteeth: effects on transport coefficients
}

\author{
V. Basiuk ${ }^{1}$, P.Huynh ${ }^{1}$, A.Merle ${ }^{2}$, S. Nowak ${ }^{3}$, O.Sauter ${ }^{2}$, JET Contributors* and the EUROfusion-IM Team** \\ ${ }^{1}$ CEA Cadarache, IRFM, 13108 St Paul Lez Durance \\ ${ }^{2}$ Swiss Plasma Center (SPC), Ecole Polytechnique Fédérale de Lausanne (EPFL), CH-1015 Lausanne, \\ Switzerland \\ ${ }^{3}$ Istituto di Fisica del Plasma IFP-CNR, 20125 Milano, Italy \\ *See the author list of "Overview of the JET results in support to ITER" by X. Litaudon et al. to be published \\ in Nuclear Fusion Special issue: overview and summary reports from the 26th Fusion Energy Conference \\ (Kyoto, Japan, 17-22 October 2016)
}

**See http://www.euro-fusionsc ${ }^{1}$ ipub.org/eu-im

E-mail: Vincent.BASIUK@cea.fr

\begin{abstract}
The neoclassical tearing modes (NTM) increase the effective heat and particle radial transport inside the plasma, leading to a flattening of the electron and ion temperature and density profiles at a given location depending on the safety factor q rational surface [0]. In burning plasma such as in ITER, this NTM-induced increased transport could reduce significantly the fusion performance and even lead to a disruption. Validating models describing the NTM-induced transport in present experiment is thus important to help quantifying this effect on future devices.

In this work, we apply an NTM model to an integrated simulation of current, heat and particle transport on JET discharges using the European Transport Simulator (ETS). In this model, the heat and particle radial transport coefficients are modified by a Gaussian function locally centered at the NTM position and characterized by a full width proportional to the island size through a constant parameter adapted to obtain the best simulations of experimental profiles. In the simulation, the NTM model is turned on at the same time as the mode is triggered in the experiment. The island evolution is itself determined by the modified Rutherford equation, using self-consistent plasma parameters determined by the transport evolution. The achieved simulation reproduces the experimental measurements within the error bars, before and during the NTM. A small discrepancy is observed on the radial location of the island due to a shift of the position of the computed $\mathrm{q}=3 / 2$ surface compared to the experimental one .To explain such small shift (up to about $12 \%$ with respect to the position observed from the experimental electron temperature profiles), sensitivity studies of the NTM location as a function of the initialization parameters are presented.
\end{abstract}

First results validate both the transport model and the transport modification calculated by the NTM model. 


\section{Introduction}

In the frame of the European Integrated Modelling effort a platform for integrated tokamak modeling has been developed, comprising complex integrated simulation workflows [1] coupling physics codes, using the graphical user friendly KEPLER software manager [2].

The main developed workflow is the European Transport Simulator (ETS) for the modeling of plasma discharges [3], embedding various interchangeable physics modules, named actors in KEPLER (see figure 1).

In this paper we present results from simulations performed with an ETS workflow adapted to study of the effect of Neoclassical Tearing Modes on diffusion equations by means of transport coefficient modifications.. The following diffusion equations are available [15]:

- Poloidal flux equation,

$\sigma_{/ /}\left[\frac{\partial}{\partial t}-\frac{\rho}{2 B_{0}} \dot{B}_{0} \frac{\partial}{\partial \rho}\right] \Psi=\frac{F^{2}}{\mu \rho B_{0}} \frac{\partial}{\partial \rho}\left[\frac{V^{\prime}}{4 \pi^{2}}\left\langle\left|\frac{\nabla \rho}{R}\right|^{2}\right\rangle \frac{1}{F} \frac{\partial \Psi}{\partial \rho}\right]+\frac{V^{\prime}}{4 \pi^{2} \rho} j_{n S}$ where $\left\langle\left|\frac{\nabla \rho}{R}\right|^{2}\right\rangle$, $\mathrm{V}^{\prime}=\left(\frac{\partial V}{\partial \rho}\right)$ are metric coefficients, $\mathrm{B} 0$ the toroidal magnetic field, $\rho$ the radial coordinate (toroidal flux), Fe diamagnetic function

- electron heat transport

$$
\frac{3}{2} \frac{\partial}{\partial t}\left(P_{e} V^{\prime^{\frac{5}{3}}}\right)+V^{\frac{2}{3}} \frac{\partial}{\partial \rho}\left[V^{\prime}(|\nabla \rho|\rangle^{2}\left(q_{e}+\lambda T_{e} \gamma_{e}\right)\right]=V^{\prime^{\frac{5}{3}}} Q_{e}
$$

Where qe is the heat source term for electrons,

$$
\begin{aligned}
& \lambda \in\left[\begin{array}{lll}
0 & \frac{3}{2} & \frac{5}{2}
\end{array}\right] \\
& \text { if } \lambda=\frac{5}{2} \text { then } Q_{\text {пео }} \approx-\frac{\gamma_{e}}{n_{e}} \frac{\partial P_{i}}{\partial \rho} \text { else } Q_{\text {пео }}=0
\end{aligned}
$$

- ion heat transport equation,

$$
\frac{3}{2} \frac{\partial}{\partial t}\left(P_{i} V^{\prime^{\frac{5}{3}}}\right)+V^{\frac{2}{3}} \frac{\partial}{\partial \rho}\left[V^{\prime}\left(|\nabla \rho|^{2}\right\rangle\left(q_{i}+\lambda T_{i} \gamma_{i}\right)\right]=V^{\prime^{\frac{5}{3}}} Q_{i}
$$

- electron density transport equation (not use in this paper)

- rotation (not use in this paper).

These equations are exactly the same equations used in an older suite of code so called CRONOS [27 28]

We focus on NTM at low poloidal (m) and toroidal (n) mode numbers The core solver in the ETS workflow (figure 2) solves the transport equations (for current, heat, particles, rotation), allowing for a direct comparison with the experimental measurements of various plasma parameters (including electron and ion kinetic profiles, self-inductance, diamagnetic energy, etc.). It integrates modules for the description of plasma 
equilibrium, neoclassical effects, sources from neutral beam and ion frequency resonance heating and finally transport processes taking into account added effects from sawteeth (ST) and NTM as well.

These latest two actors are implemented in the ETS workflow for the description of the corresponding instabilities, namely:

- a sawtooth module includes a numerical procedure for the modeling of the effect of individual sawtooth crashes on the plasma temperature, density and current profiles.. The sawtooth model is based on the original model [4] and slightly modified [5]. It determines when a sawtooth crash is triggered, when the shear at $\mathrm{q}=1$ exceeds a critical shear. Full reconnection is used in this work to determine the new profiles. The ETS evolves these profiles up to the next sawtooth crash

- an NTM module from the NTMwf code [6] modeling the NTM size [7] and frequency [8] evolution and the modification of the perpendicular radial electron/ion temperature and density transport diffusion coefficients $\chi_{\perp}$ around the mode location corresponding to the safety factor $\mathrm{q}=\mathrm{m} / \mathrm{n}$ position [9]. The magnetic island leads to a flattening of the kinetic profile, hence an increased radial transport which in turn increases the island width through the perturbed bootstrap current. Note that "anomalous" (turbulent) transport is observed to decrease inside the island [10], but this is due to the reduction of the local gradients, and thus of the main drive for microinstabilities.

The paper is organized as follows: first the sawteeth and NTM models are presented, as well as their implementation in the transport simulator and then used to simulate the time evolution of JET plasma discharges where $3 / 2$ and 5/4 NTMs have been observed, in order to analyze the effects on the time evolution of the electron temperature profile in particular.

\section{Description of numerical tools}

\subsection{ETS workflow}

The ETS workflow, developed within the European EFDA Task Force on Integrated Tokamak Modelling [1], integrates many physics codes to perform a complete simulation of plasma confined in a tokamak. Among those, and used for this paper, CHEASE [11] (fixed boundary equilibrium code), NCLASS [12] (neoclassical transport code), NEMO/SPOT [13-14] (neutral beam/ion frequency resonance heating codes), and the transport models Kiauto [15] (simple model based on a scaling law for the energy confinement time) for the transport in the pedestal and Bohm/gyroBohm [16] for the core transport(figure 2). The integration is achieved by adapting the codes to exchange data in the form of a standardized data structure and creating KEPLER actors using ITM tools [3]. In the workflow the four transport equations for the poloidal flux, the electron and ion temperature and the plasma density are solved. It is able to take into account instantaneous events such as sawteeth and abnormal events such as neoclassical tearing modes, consequently modifying the internal time step and transport coefficients (both in energy and particle channels). The numerical scheme is based on finite differences in space and an implicit method in time. 
For the simulation of a plasma discharge, the ETS workflow is used in a first phase in its interpretative mode, where only the current diffusion equation is solved and the kinetic profiles are fitted using the reconstruction of the magnetic equilibrium. During this phase a more precise 2D equilibrium is computed modifying the fit of the kinetic profiles and performing a new run of the workflow. One iteration is sufficient to obtain a good "kinetic" equilibrium.

In a second phase, the transport coefficients are adapted to reproduce the background electron temperature without NTM. Note that the aim of this paper is not to discuss the background transport simulation but to test the effects of ST and NTMs on self-consistent transport simulations and on the kinetic profiles. All the source terms (non-inductive current from NBI and neoclassical bootstrap, equipartition, radiation, ohmic power) can be computed by means of modules embedded in the ETS workflow.

Finally, in a third phase, both ST and NTM modules are activated in the workflow for a full plasma discharge simulation (computing all the kinetic profiles except the ion density, which is prescribed). The present workflow contains 5 species fully stripped and derived from 5 equations: the electron density, the charge effective profile and the ratios $n_{H} / n_{D}, n_{D} / n_{e}, n_{C} / n_{O}$, where $n_{H}$ is the hydrogen density, $n_{B}$ the deuterium density, $n_{C}$ the carbon density $n_{O}$ the oxygen density and all species share the same profile.

\subsection{Sawteeth model}

The sawteeth model described in this work is based on the original article by Porcelli [4] with some modifications for regimes where the electron temperature exceeds the ion temperature as found in the paper by Sauter [5]. This approach has originally been validated using the PRETOR code for NBI-stabilized sawteeth in JET [17] and for EC-heated discharges in TCV [18].

The model has two major components. The first one is a set of criteria for the triggering of a sawtooth crash. The equations for the crash criteria are as follows:

$$
\begin{aligned}
& { }_{-} \delta W_{\text {core }}>c_{h} \omega_{D h} \tau_{A} \\
& \_\delta W_{\text {tot }}>\frac{1}{2} c_{\text {mod }} \omega_{* i} \tau_{A} \\
& C_{\rho} \hat{\rho}<-\delta W_{\text {tot }}<\frac{1}{2} c_{\text {mod }} \omega_{* i} \tau_{A} \\
& \max \left(\gamma_{\text {res }}\right)>c_{*} \sqrt{\omega_{* i} \omega_{* e}}
\end{aligned}
$$

with $\delta W_{\text {core }}=W_{M H D}+\delta W_{K O}$ and $\delta W_{\text {tot }}=W_{\text {core }}+\delta W_{\text {fast }}$,

where $\omega *$ (resp. ${ }^{*}$ ) is the ion (resp. electron) diamagnetic frequency.

If one of the first two criteria $(1.1,1.2)$ is satisfied, then the crash is triggered by the ideal internal kink mode. The first equation (1.1) corresponds to the case of an unstable ideal mode where the influence of fast particles is negligible. The second equation (1.2) treats the case of an unstable ideal mode with significant contribution from the fast particles. The third criteria $(1.3 \mathrm{a}, \mathrm{b})$ correspond to a crash triggered by the resistive internal kink where effects in the resonant layer dominate. Both inequalities (1.3a) and (1.3b) need to be 
satisfied to trigger sawtooth crash. In the model, $c_{*}, c_{h}, c_{m o d}, c_{\rho}$ are free parameters of order unity. Since the model uses simple formulas to compute the different growth rates, these free parameters are there to account for missing physics such as the effect of geometry or non-linearities. Their value must be adjusted for each simulation but they are mostly scenario dependent. Moreover for a particular scenario usually a single criterion is satisfied and is responsible for the sawtooth crashes. This means that effectively only one free parameter needs to be adjusted. To simulate past experiments, this parameter can be chosen to match the experimental sawtooth period from a single phase such as the ohmic phase at the start of the experiment. The parameter is kept constant during the simulation.

The second component is a set of transformation rules for determining the post-crash profiles. The sawtooth module implements two different sets of transformation rule for the plasma profiles in the event of a sawtooth crash. The first one corresponds to the Kadomtsev complete reconnection model [19]. This model relies on the assumption that surfaces of equal helical flux coalesce while conserving the total number of particles and total energy.. The second model corresponds to an incomplete reconnection of the volume inside the $\mathrm{q}=1$ surface. The resulting q-profile is piece-wise linear in the reconnecting region while the density and temperature profiles are assumed to be flat inside the mixing radius while the global number of particles and energy is conserved. A complete description of this incomplete reconnection model can be found in [4]. The Kadomtsev model is used in our simulations for all simulated species (electrons, main ions and impurities) affected by the sawtooth crash. This choice is only motivated by the good agreement of our simulations with the experiments in terms of sawtooth period and size of the region affected by the crash. More simulations should be performed in order to assess which model is best to reproduce accurately the reconnection events during sawteeth in tokamaks.

1.5 D transport codes must also periodically compute a full 2D equilibrium via a Grad-Shafranov solver. Since it can affect a large fraction of the plasma volume and can induce large variations in the current and pressure profiles, a sawtooth crash requires that the $2 \mathrm{D}$ equilibrium is re-computed immediately afterwards. In particular our model ensures that the q-profile in the ETS iteration following the crash is consistent with the one given by our reconnection model. The evolution between consecutive sawtooth crashes is determined by the transport model and solver to which it is coupled, in this case the European Transport Solver. This model has been also included in other transport codes [20] using the same code base and changing only the interface.

\subsection{NTM module}

The NTM module (referring to the "NTMwf" code) models the NTM dynamics in terms of width (w) and frequency $(\omega)$ time evolution taking into account the basic physics contribution and self-consistent effects on transport for which it has been integrated in the ETS workflow. A Generalized Rutherford Equation (GRE) describing the mode amplitude evolution is written as $[8,9]$ : 


$$
\begin{aligned}
g_{1} \frac{\tau_{R}}{r_{s}} \frac{d w}{d t}= & r_{s}\left[\Delta_{0}^{\prime}+\Delta_{b s}^{\prime}-\Delta_{G G J}^{\prime}-\Delta_{p o l}^{\prime}-\mathfrak{R e}\left(\Delta_{w}^{\prime}\right)\right]= \\
= & r_{s}\left[\Delta_{0}^{\prime}+a_{b s} \Delta_{b s_{0}}\left(\frac{f_{b s e} w}{w^{2}+w_{d, e}^{2}}+\frac{\left(1-f_{b s e}\right) w}{w^{2}+w_{d, i}^{2}}\right)\right. \\
& \left.-a_{g g j} \Delta_{G G J_{0}} \frac{1}{\sqrt{W^{2}+0.2 w_{d, e}^{2}}}-a_{p o l} \Delta_{p o l} \frac{\rho_{\theta i}^{2} w}{W^{4}+w_{d, p o l}^{4}}-\mathfrak{R e}\left(\Delta_{w}\right) \frac{1}{r_{s}}\right]
\end{aligned}
$$

where:

$$
\Delta_{0}^{\prime}=-m / r_{s} ; \quad \Delta_{b s_{0}}=\beta_{p} \sqrt{\varepsilon}\left|\frac{L_{q}}{L_{p}}\right| ; \quad \Delta_{G G J_{0}}=\beta_{p} \varepsilon^{2} \frac{L_{q}}{r_{s}} \frac{L_{q}}{\left|L_{p}\right|}\left(1-1 / q^{2}\right)
$$

$$
\Delta_{p o l_{0}}=\beta_{p}\left(\frac{L_{q}}{L_{p}}\right)^{2} g\left(\varepsilon, \boldsymbol{v}_{i i}\right) \frac{\omega\left(\omega_{*_{i}}-\omega\right)}{\omega_{*_{e}}^{2}} ; \quad \Delta_{w}=2 m\left(\frac{r_{s}}{d}\right)^{2 m} \frac{\left(\boldsymbol{\tau}_{w} \omega\right)^{2}+i\left(\boldsymbol{\tau}_{w} \omega\right)}{\left(1+\left(\boldsymbol{\tau}_{w} \omega\right)^{2}\right)}
$$

The equation includes the usual tearing stability parameter $\Delta^{\prime}{ }_{0}$ due to the equilibrium current gradient [22], the destabilizing $\Delta^{\prime}{ }_{\text {bs }}$ term originated by the perturbed bootstrap current $[8,23]$, the stabilizing term $\Delta^{\prime}{ }_{\text {ggj }}$ due to the magnetic field curvature $[8,24]$, the ion polarization current $\Delta_{\text {pol }}^{\prime}$ term depending on the mode frequency $[8,25]$ and the stabilizing $\Delta^{\prime}$ wall due to eddy current in the resistive wall [26]. The $\Delta^{\prime}{ }_{\text {pol }}$ can be destabilizing if the mode rotates in the electron drift direction or with a frequency larger than the ion drift. In GRE $w_{d, e}$ is the small width due to finite perpendicular transport, $w_{d, i}$ the width proportional to the ion banana width, $f_{b s e}$ the fraction of the electron contribution to the bootstrap, $w_{d, p o l}=\rho_{\theta i}$ the poloidal ion Larmor radius, $m$ the poloidal mode number, $r_{s}$ the mode radial location, $\tau_{R}$ the resistive time, $g_{l}$ a numerical factor $=0.82, \beta_{p}$ the poloidal beta, $\varepsilon$ the inverse aspect ratio, $L_{q}$ and $L_{p}$ the safety factor and pressure gradient scale lengths, $\omega *_{\mathrm{e}}$ and $\omega *_{\mathrm{I}}$ the electron and ion diamagnetic frequencies, $g$ a factor depending on collisionality, $d$ the radius of the vacuum vessel and $\tau_{w}$ the resistive wall time.

The frequency equation includes effects from the braking electromagnetic torque due to the eddy currents, the viscous drag with anomalous viscosity and the inertial braking due to a growing moment of inertia. The phase equation is considered as well [9]:

$$
\frac{d}{d t} \frac{d \phi}{d t}=\frac{d \omega}{d t}=\frac{1}{I_{\phi}}\left[-n\left(T_{\phi e m}+T_{\phi v i s c}\right)-T_{\phi i n}\right] ; \quad \frac{d \phi}{d t}=\omega
$$

with 


$$
\begin{aligned}
& T_{\phi e m} \propto R r_{s} / \mu_{0}\left[B r_{s} / 16 R q L_{q}\right]^{2} \operatorname{I} m\left(\Delta_{w}^{\prime}\right) W^{4} \\
& T_{\phi v i s c} \propto r_{s}^{2} R \rho_{i}\left(\omega-\omega_{* i}\right) /\left(w+w_{\text {visc }}\right) \tau_{M} \\
& T_{\phi i n} \propto\left(\omega-\omega_{* i}\right) d l_{\phi} / d t
\end{aligned}
$$

and where $r_{i}$ is the ion mass density, $w_{\text {visc }}$ a small width to avoid numerical problems, $t_{M}$ is the time associated to the damping rate and $\mathrm{I}_{\mathrm{f}}$ the moment of inertia of plasma.

From Eq. (1.6) it can be seen that the mode rotation is progressively reduced by the electromagnetic braking as the island size increases and lowered by the inertial one at small initial island width. The viscous torque decreases as the mode width increases, but it can change the slope of the frequency evolution if the sign of the difference between the mode and ion diamagnetic frequencies changes sign.

Evaluations of the effects of the NTM on transport are done including the equations (1.4) and (1.5) in the ETS workflow and computing additional transport coefficients based on the mode characteristics (location and width).

The NTM module is integrated in the transport convergence loop of the ETS workflow and includes three actors: the first (checktearing) checks if the conditions for an NTM seed island or tearing mode destabilization are met, the second (ntmets) calculates the size and the frequency evolution of the mode, the third (ntmDeff_ets) evaluates the changes of transport coefficients.

The sawteeth module, providing the sawtooth periods, is linked to the checktearing actor in which the time onset of NTM can be fixed at a sawteeth crash time after a long ST period, for example, since this is an important triggering mechanism [27-29]. It can also be triggered if the value of $\Delta^{\prime}$ exceeds a threshold. In a first stage, for the sake of simplicity, $\Delta^{\prime}$ can be computed from cylindrical approximation (neglecting toroidal effects) $[27,30]$, but in a later stage more reliable calculations from a full 3D MHD code will be provided. Note that we do not rely on this value in this paper, since the mode is always relatvely large (including the seed island due to sawtooth crash), thus we use $\mathrm{r}_{\mathrm{s}} \Delta_{0}^{\prime}=-\mathrm{m}$ (Eq.(1.4)).

The modification of the transport coefficients is modelled by changing the perpendicular electron/ion temperature and density transport diffusion coefficients $\chi_{\perp}$ around the mode location at the minor radius $r_{s}$ where the safety factor $\mathrm{q}=\mathrm{m} / \mathrm{n}$. These coefficients are updated by adding a Gaussian perturbation to the unperturbed ones [10]:

$$
\chi_{\perp, \text { updated }}=\chi_{\perp, \text { unperturbed }}+\chi_{\perp, w} \quad \text { where } \quad \chi_{\perp, w}=c_{\chi} \chi_{\perp, \text { unperturbed }} \exp \left(-\left(2\left(r-r_{s}\right) /\left(c_{w} w\right)\right)^{2}\right)
$$

The $\mathrm{c}_{\chi}$ and $\mathrm{c}_{\mathrm{w}}$ coefficients allow changing the amplitude and the width of the perturbation, respectively. Usually, $\mathrm{c}_{\chi} \sim 10-20$ and $\mathrm{c}_{\mathrm{w}} \sim 1$.

This $\chi_{\perp}$ modification allows simulating the flattening of the experimental temperature and density profiles around the mode location due to the increased perpendicular radial transport. The ETS assumes that 
local transport can be considered with the radial diffusion coefficient being composed additively by the neoclassical contribution, "anomalous" (from microinstabilities) and from macroscopic MHD modes.

\section{NTM behavior simulation in JET discharge}

\subsection{Analysis description}

The effects of NTMs on the transport coefficients are analyzed for some JET discharges. Particularly, two shots are considered coming from the JET database (figure 3):

- a first discharge (\#76791) at |1.6| MA of plasma current and $2 \mathrm{~T}$ of magnetic field with 20 MW of neutral beam injection (NBI) to heat the plasma in H-mode (high confinement) (see figure 3a, 5a),

- a second one (\#86535) at higher plasma current and magnetic field (|2.5 MA|, 2.36 T) with both $20 \mathrm{MW}$ of NBI and $2 \mathrm{MW}$ of ICRH (Ion Cyclotron Resonance Heating) and high level of radiation and some impurity accumulation (see figure $3 b 5 b$ ).

In the first shot a $3 / 2$ NTM is excited at $49.6 \mathrm{~s}$ at a sawtooth crash after a long sawtooth period during the H-mode phase and the mode lasts for about $2 \mathrm{sec}$, while in the latter a 5/4 NTM appears in H-mode at 47.1s also after a very long sawtooth period and stays for about $3 \mathrm{sec}$ (figure 4).

We have developed a protocol in ETS adapted to the study of JET shots. In the initialization phase we use both the super heterodyne diagnostic (namely c"KK3" in JET language)) and the High Resolution Thomson Scattering diagnostic (HRTS) to fit the electron temperature mapped on a plasma equilibrium reconstruction using magnetic measurements (EFIT). For the density, we take into account only HRTS but we reconstruct the interferometry chord and compare with the experiment for validation.

In the first interpretative simulation of the shot, where only the current diffusion equation is solved, a new equilibrium is recalculated taking into account the boundary condition for the poloidal flux diffusion equation provided by the measured plasma current. However, in order to obtain a more accurate fit of the electron and ion temperature a new magnetic fixed boundary equilibrium calculation is performed using the module CHEASE [11] in the ETS workflow. This second reconstructed equilibrium is used for a new interpretative run to obtain a more reliable reconstruction of the safety factor profiles for the NTM location as a function of normalized toroidal flux. However, a slight discrepancy of the mode radial location $(\leq 15 \%)$ is observed comparing the different equilibrium reconstructions (figure 5). This is expected since EFIT has no adequate internal constraints and obtains $\mathrm{q}_{\min }>1$, although the discharge is sawtoothing.

In the second phase a complete predictive run (achieved in a few days of CPU time) solves the four transport equations (poloidal flux, electron and ion temperature, electron density) and includes all the source terms (NBI non inductive current, neoclassical bootstrap current, equipartition, radiation, and ohmic power) computed using the corresponding physics module actors integrated in the ETS workflow. Particularly, the NBI heating and current drive are provided with the modules NEMO and SPOT whereas the bootstrap current 
with the code NCLASS. It should be noted that for discharges with ICRH we do not compute the corresponding power deposition since its contribution, in particular to the $\mathrm{q}$ and $\mathrm{T}_{\mathrm{e}}$ profiles, is small.

The transport coefficients are adapted to fit the experimental profile without the NTM effects. A Bohm/gyroBohm model [16] is used to fit the profiles in the central part of the plasma up to the pedestal top in H-mode, while a scaling law model Kiauto [15] is applied to compute the pedestal in the H-mode phase.

Finally, in a third phase both ST and NTM modules are activated to the workflow for a fully predictive plasma discharge simulation where the transport coefficients are modified accordingly to simulate the effect of the tearing modes on the profiles. The model described in the previous section has been used for updating the transport coefficients.

\subsection{Simulation of JET \#76791}

We show and discuss the simulation of experimental electron temperature profiles for the JET discharge \#76791 in the high confinement scenario with NBI heating. The electron density profiles from HRTS diagnostic and their fits are shown for times before and after the $3 / 2$ onset (figure 6). For this discharge the electron density profiles are prescribed and no recalculation by the ETS is provided, in order to better test the effects of NTMs on the Te profile, which is the better diagnosed kinetic profile modified by magnetic islands. Electron density profiles, measured by a high resolution Thomson Scattering (HRTS) diagnostics and prescribed in ETS, are shown in Fig. 6 for one time before the mode onset (48 s) and three times after the mode appearance $(49.9 \mathrm{~s}, 50.5 \mathrm{~s}$ and $50.7 \mathrm{~s})$. The profiles in ETS marginally fit the experimental data at the plasma edge, while they are in very good agreement for the last 2 times. Electron temperature profiles, measured by electron cyclotron emission (ECE) diagnostics and HRTS, and recalculated by the ETS in the second predictive phase without NTM, are shown for the same time as in figure 6 (figure 7). We note that typical flattening around the mode location during the NTM appearance is observed on the $T_{e}$ profile, while no clear evidence is seen on $n_{\mathrm{e}}$ profiles from HRTS diagnostic, although the profile is relatively flat in any case.

The comparison of the measured and predictive calculated time traces of the main global parameters are shown in figure 8 . The experimental quantities are provided by the EFIT code, while the simulated ones are obtained from the second reconstructed equilibrium in predictive mode. The agreement is between 6-30\%. Using the profiles saved in the standardized interface CPOs [3] during the predictive run, the full discharge is simulated adding the ST and NTM modules in the ETS workflow.

Concerning the simulation of sawteeth, the assumption was made that the main triggering mechanism was the resistive internal kink mode, since the fast particle contribution is relatively small in these discharges. Hence, a scan in the $c_{*}$ parameter was performed. As equation (1.3) is equivalent to a condition of the form $s_{1}>s_{1, \text { crit }}$ where $s_{1}$ is the magnetic shear at the $\mathrm{q}=1$ surface, we have plotted in figure 9 a the sawtooth period around the time of the NTM onset $(49.7 \mathrm{~s})$ as a function of $s_{1, \text { crit }}$. As $s_{1, \text { crit }}$ increases, the sawtooth period increases as well and for $s_{1, c r i t} \cong 0.2$ the experimental period of about $350 \mathrm{~ms}$ was obtained. For the corresponding value of $c_{*}$, we show in figure $9 \mathrm{~b}$ the evolution of the central values for the quantities, $q, T_{e}$ and 
$\mathrm{T}_{\mathrm{i}}$. It is apparent that the sawtooth period increases with time before the NTM onset (around $49.7 \mathrm{~s}$ experimentally). This allowed us to link in the simulation the onset of the $3 / 2$ NTM with the sawtooth period exceeding a certain value as we already noted that experimentally the NTM onset can be linked to sawteeth of sufficient duration.

In figure 10 the mode size and frequency evolution are shown. The relative evolution of the calculated mode frequency (in the plasma frame) is found nearly similar, at least up to the H-L transition, to the measured one (in the laboratory frame) taking into account the three physical effects due to the electromagnetic, viscous and inertial torques. The oscillations around the experimental frequency trace are probably due to the change in sign of the difference $\left(\omega-\omega *_{\mathrm{i}}\right)$ (see Eq. (1.6)). In figure 11 the comparison of the experimental (only from ECE measurements) and calculated electron temperature profiles are plotted for the three time slices after the NTM considered in figure 6. The mode sizes from the computed flattening of the profiles look in nearly agreement with the experimental ones. In the simulations the electron diffusion coefficients have been modified with a perturbation centered at the mode location characterized by a Gaussian function (Eq. (1.7)) with $\mathrm{c}_{\chi, \mathrm{Te}}=20$ and $\mathrm{c}_{\mathrm{w}, \mathrm{Te}}=1$.

The saturated width of the $3 / 2$ mode is about $0.12 \mathrm{~m}$ in good agreement with the experimental value coming from the size of the flattened portion of the $\mathrm{T}_{\mathrm{e}}$ profile. A slight shift of the mode location $(\sim 5-15 \%)$ between measured and calculated profiles is due to the uncertainties of the q profile, reconstructed with a lack of internal constraints by EFIT code. The flattening on the electron temperature in the NTM phase is reproduced modifying the electron transport coefficient by a Gaussian function (see Eq. (1.7) centered at the $\mathrm{q}=3 / 2$ location with $\mathrm{c}_{\chi, \mathrm{Te}}=20$ and $\mathrm{c}_{\mathrm{w}, \mathrm{Te}}=1$ (similarly for $\mathrm{T}_{\mathrm{i}}$ ). The perpendicular electron transport coefficient profiles are plotted in figure 12 with the corresponding $\mathrm{T}_{\mathrm{e}}$ and q profiles at $\mathrm{t}=48 \mathrm{~s}$ before the mode onset and $\mathrm{t}=50.7 \mathrm{~s}$ after the mode appearance when the Gaussian perturbation, described above, has been considered.

The values of the coefficients $\mathrm{c}_{\mathrm{w}, \mathrm{Te}}$ and $\mathrm{c}_{\chi, \mathrm{Te}}$ used in the simulations have been determined by the analysis of the effects of the Gaussian perturbation on $T_{e}$ flattening changing the transport coefficients. In figure 13 the effects of the Gaussian perturbation on electron temperature and diffusion coefficient profiles are shown changing $c_{\chi, T e}$ for a constant $c_{w, T e}$ and with a smaller $c_{w, T e}$. First we look in figure 13(left) at the radial derivative of the temperature profile. We see that with $\mathrm{c}_{\chi, \mathrm{Te}}=10$, the profile is not flattened enough (local derivative about 2 times larger than the experimental data), while with $\mathrm{c}_{\chi, \mathrm{Te}}=20$ or more then $\mathrm{dT}_{\mathrm{e}} / \mathrm{d}$ is similar to the experiment and more importantly it does not depend on the value of $c_{\chi}$,Te anymore. In figure 13 (right) we look at the shifted dTe/d $\rho$ profiles, to match the local maxima, in order to better see the effect of $\mathrm{c}_{\mathrm{w}, \mathrm{Te}}$. We see that $c_{w, T e}=0.5$ is too small. Therefore we propose $c_{w, T e}=1.0$, that is the Gaussian width is directly proportional to the island width, and $\mathrm{c}_{\chi, \mathrm{Te}}=10$ as default values. With $\mathrm{c}_{\chi, \mathrm{Te}} \geq 20$ the results do not change too much. This is checked by zooming the profiles near the rational surface, figure 14(left). We see that the main effect of the NTM is indeed to "shift" the gradient region of $T_{e}$ with a flattened zone due to the island width. This shift is well reproduced with $\mathrm{c}_{\mathrm{w}, \mathrm{Te}}=1$ and $\mathrm{c}_{\chi, \mathrm{Te}}=10$, even if the exact location is not reproduced due to 
small differences in the q profile, as previously mentioned. This also confirms the basics of the belt model leading to a global confinement degradation due to the island proportional to the island width [25].

A comparison in $3 \mathrm{D}$ and $2 \mathrm{D}$ plots of the electron temperatures without and with the NTM modelling is shown in a time interval between $48 \mathrm{~s}<50.5 \mathrm{~s}$ (figures 15-16). The modified shape in the interval time of the observed $3 / 2$ mode is due the changes of the electron transport coefficients, as described above.

\subsection{Simulation of JET \#86535}

The effect of the NTM on the profiles is presented for a second JET shot \#86535 with an observed 5/4 mode for about $3 \mathrm{~s}$ from $\mathrm{t}=47.1 \mathrm{~s}$ in the H-mode with NBI and ICRH heating. This shot presents also a $1 / 1$ and $2 / 2$ mode in the same time interval. The comparison of the measured and predictive calculated time traces of the main global parameters are shown in figure 17. The experimental quantities are provided again by the EFIT code, while the simulated ones are obtained from the second reconstructed equilibrium in predictive mode. The ICRH power deposition is not included for simplification and because its contribution is also small. Experimental and calculated electron temperature profiles without the NTM modelling are shown in figure 18. The same assumption of crashes driven by resistive kink modes was made and a similar procedure was applied. The free parameter was adjusted to match the simulated sawtooth period to the experimental one in the ohmic L-mode phase at the beginning of the discharge. The experimental sawtooth period in this phase was estimated to be around $50 \mathrm{~ms}$. As the additional power is switched on, the temperature rises and the resistive time increases resulting in longer sawteeth. In our simulation we obtain a sawtooth period of about $800 \mathrm{~ms}$ at $\mathrm{t}=47 \mathrm{~s}$ which corresponds to the onset of the NTM in reasonable agreement with the experimental value as can be seen in figure 19.

The comparison between measured (from super heterodyne diagnostic)) and simulated $T_{e}$ profiles in presence of the 5/4 mode is plotted in figure 20. A shift of the mode location is also found in the calculations. The flattening on the electron temperature due to the NTM is well reproduced updating the electron transport coefficients by a Gaussian function centered at the $q=5 / 4$ location with $c_{\chi, T e}=10$ and $c_{w}=1$. The perpendicular electron transport coefficient profiles, modified by the Gaussian perturbation, are plotted in figure 21 with the corresponding $\mathrm{T}_{\mathrm{e}}$ and q profiles for 2 times before $(46.5 \mathrm{~s})$ and after $(48 \mathrm{~s})$ the NTM onset, as done in Fig.12 for the previous discharge 76791. Similar sensitivity study, as in Fig. 13, have been performed for this shot leading to the same conclusions for the coefficient $\mathrm{c}_{\mathrm{w}}=1$, while the coefficient $\mathrm{c}_{\chi, \mathrm{Te}}=$ 10 has been considered to best fit the experimental measurements. The simulations with $c_{\chi, T e} \geq 20$ are not sensitive to these values anymore, and does not need to be adapted.

A comparison in $3 \mathrm{D}$ and $2 \mathrm{D}$ plots of the electron temperatures without and with the NTM modelling is shown in a time interval between $47 \mathrm{~s}<49$ s (figures $22-23$ ). 


\section{Conclusions}

We have addressed in this paper two main points, a physical one where we reproduce the effect of sawteeth and NTM on the kinetic profiles (ion/electron density and temperature) and the use of the ETS workflow demonstrating a sophisticated integrated simulation. The NTM model has been shown to reproduce the experimental observations with the main effects captured by the generalized Rutherford equation, solved in parallel to the ETS kinetic profile evolution, and the effect of the island of a given width on transport, selfconsistently incorporated within the ETS workflow.

The triggering due to an increasing sawtooth period has been simulated as well. The models can easily be coupled to other transport solvers.

This result is of great interest to study the control of NTMs in ITER, using both additional heating and first principal transport model (GLF23 for instance). Indeed the present results show that a self-consistent simulations of the effects of heating and current drive on the NTMs, which will be included in the generalized Rutherford equation, coupled with the effects of the NTM on the transport equation can be obtained with a workflow as described in this paper. Moreover, the profiles modified by the NTMs are then used to compute the heating and current drive deposition, leading to a fully self-consistent simulation.

\section{Acknowledgements}

This work has been carried out within the framework of the EUROfusion Consortium and has received funding from the EURATOM research and training programmed 2014-2018 under grant agreement No 633053. The views and opinions expressed herein do not necessarily reflect those of the European Commission. Antoine Merle and Olivier Sauter have been supported in part by the Swiss National Science Foundation.

\section{References}

[0] Hegna C C and Callen J D, Phys. Plasmas 4, 2940 (1997)

[1] Falchetto G et al 2014 Nucl. Fusion 54043018

[2] https://kepler-project.org

[3] Imbeaux F et al 2010 Comput. Phys. Commun. 181987

[4] Porcelli F et al 1996 Plasma Phys. Control. Fusion 382163

[5] Sauter O et al 1999 Theory of Fusion Plasmas (Proc. Joint Varenna-Lausanne Int. Workshop)

(Varenna, 1998) ed J W Connor, E Sindoni and J Vaclavik, ISPP-18 (Bologna: Editrice Compositori) 403

[6] Sauter O et al 2017 paper to be submitted to Comput. Phys. Commun.

[7] Sauter O et al 2002 Plasma Phys. Control. Fusion 441999

[8] Ramponi G et al 1999 Phys. Plasmas 63561

[9] Turri et al 2008 in Proc. 22 ${ }^{\text {nd }}$ IAEA Fusion Energy Conf. (FEC), Geneva paper EX/P3-6

[10] Poli E et al 2010 Plasma Phys. Control. Fusion 52124021

[11] Lütjens H et al 1996 Comput. Phys. Commun. 97219

[12] Houlberg W A etal 1997 Phys. Plasmas 43230

[13] Schneider M et al 2005 Plasma Phys. Control. Fusion 472087

[14] Schneider M et al 2011 Nucl. Fusion 51063019

[15] Artaud J F et al 2010 Nucl. Fusion 50043001 
[16] Erba M et al 1998 Nucl. Fusion 381013

[17] Angioni C et al 2002 Plasma Phys. Control. Fusion 44205

[18] Angioni C et al 2003 Nucl. Fusion 43455

[19] Kadomtsev B B 1975 Sov. J. Plasma Phys. 1389

[20] Kim D et al 2016 Plasma Phys. Control. Fusion 5855002

[21] Reimerdes H et al 2002 Phys. Rev. Lett. 88105005

[22] Chapman I et al 2010 Nucl. Fusion 50102001

[23] Canal G P et al 2013 Nucl. Fusion 53113026

[24] Lazzaro E et al 2015 Nucl. Fusion 55093031

[25] Chang Z et al 1990 Nucl. Fusion 2219

[26] Altintas I et al 2004 Proceedings of the 16th International Conference on Scientific and Statistical Database Management 423-424

[27] Giovannozzi E et al 2014 in Proc. of $41^{\text {th }}$ EPS Conference on Plasma Physics $\mathbf{P 1 . 0 1 5}$

[28] V. Basiuk et al, Nuclear Fusion, Volume 43 822, Number 9

[29] F Imbeaux and Y Peysson 2005 Plasma Physics and Controlled Fusion 472041 


\section{EUROPEAN TRANSPORT SOLVER}

Workflow parameters

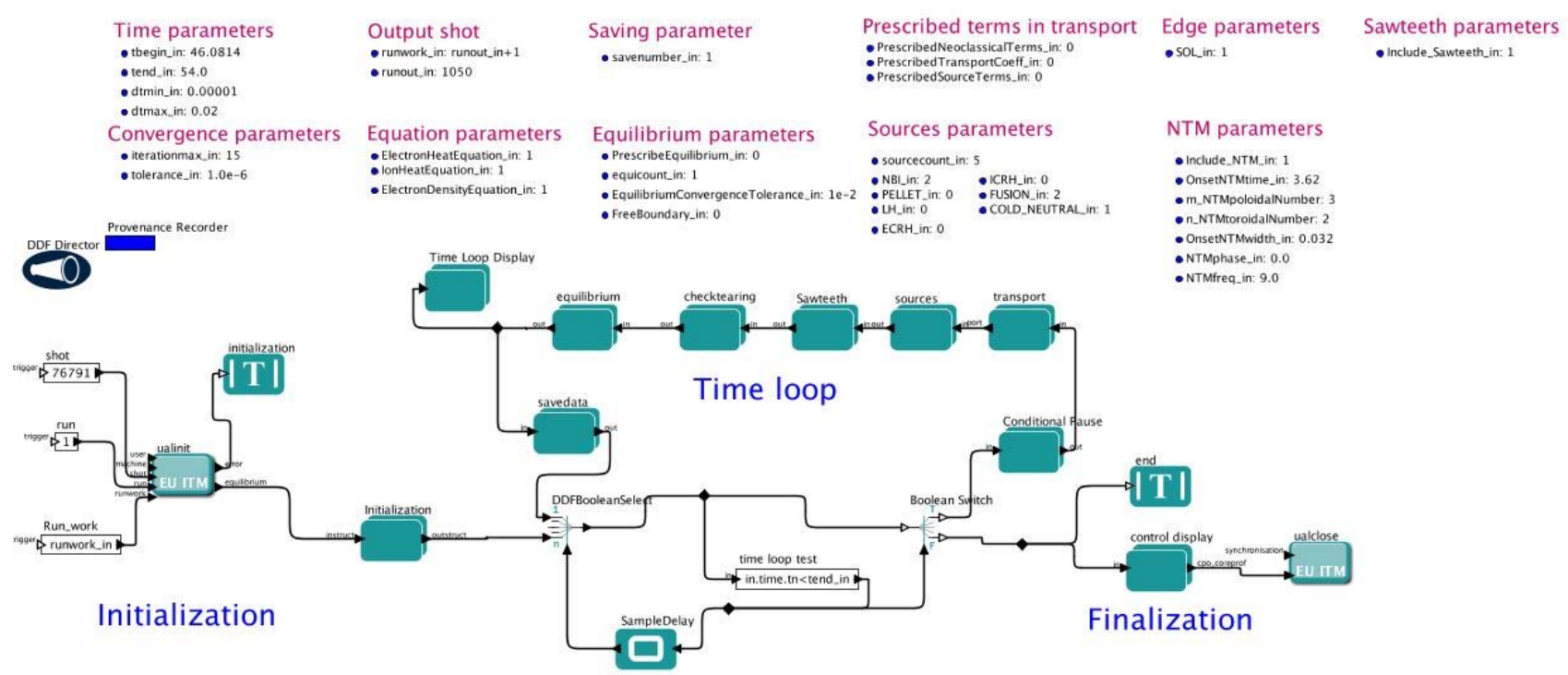

Figure 1. Top level view of the ETS workflow, written with the graphical task scheduler tool, KEPLER. Each box (composite actor) can contains sub-workflows which is the case of additional heating modules. 


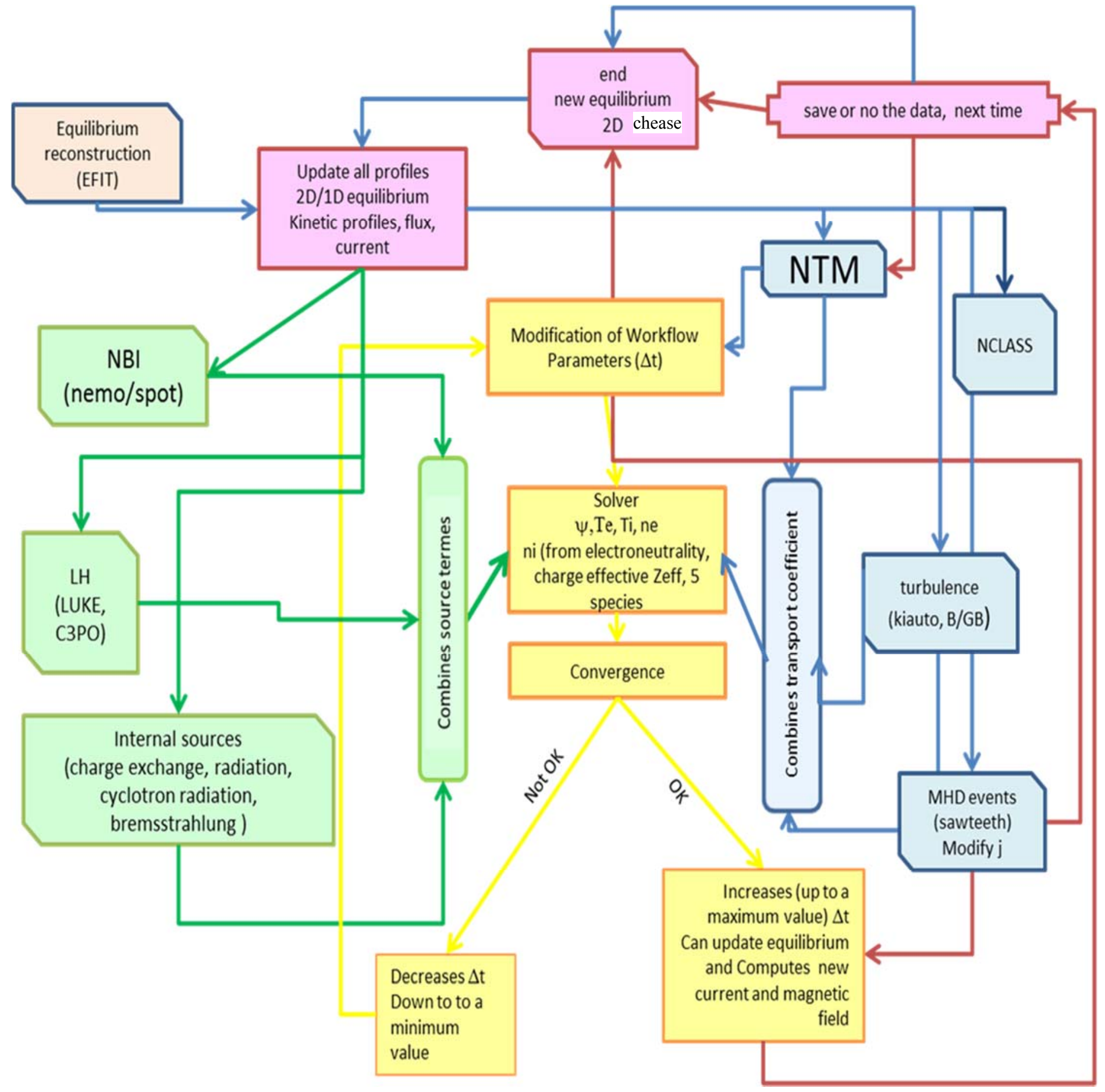

Figure 2. Schematic view of a workflow. Yellow boxes are devoted to the heart of the tool (solving the transport equations). Green boxes concern the computation of the source terms (external such as NBI heating or internal such as bremsstrahlung radiation), blue boxes for the transport model and pink for the internal loop (time loop, equilibrium loop to have coherence between the poloidal flux from the current diffusion equation and from the equilibrium solver). 


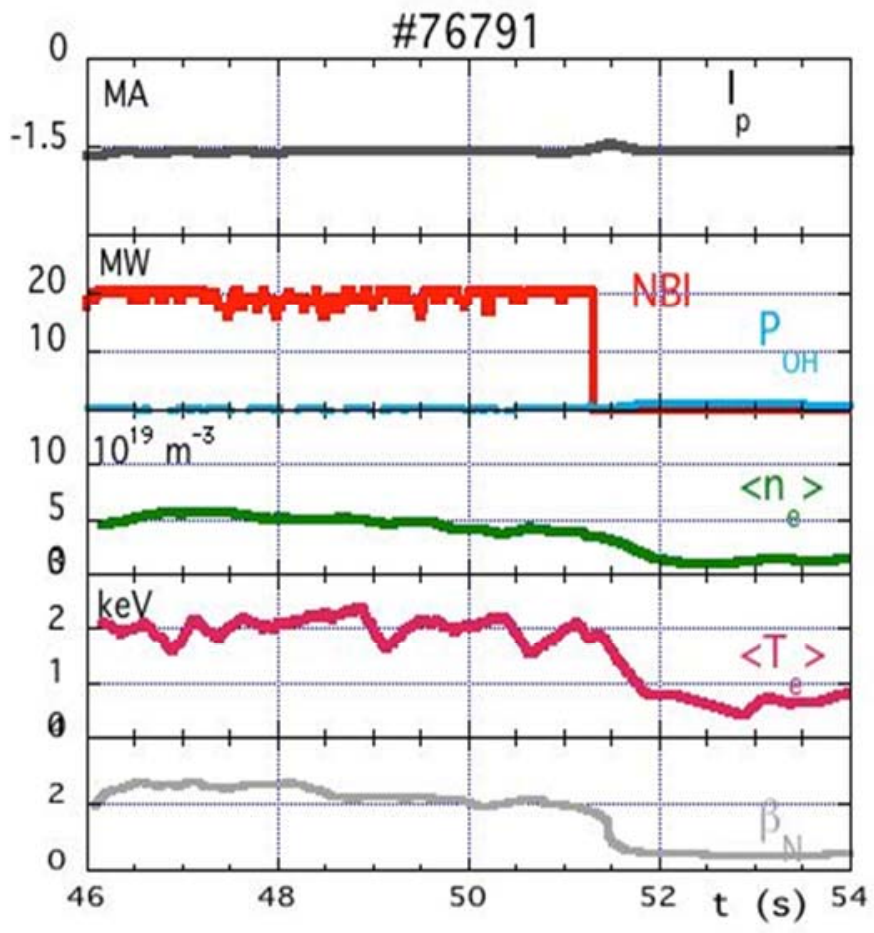

(a)

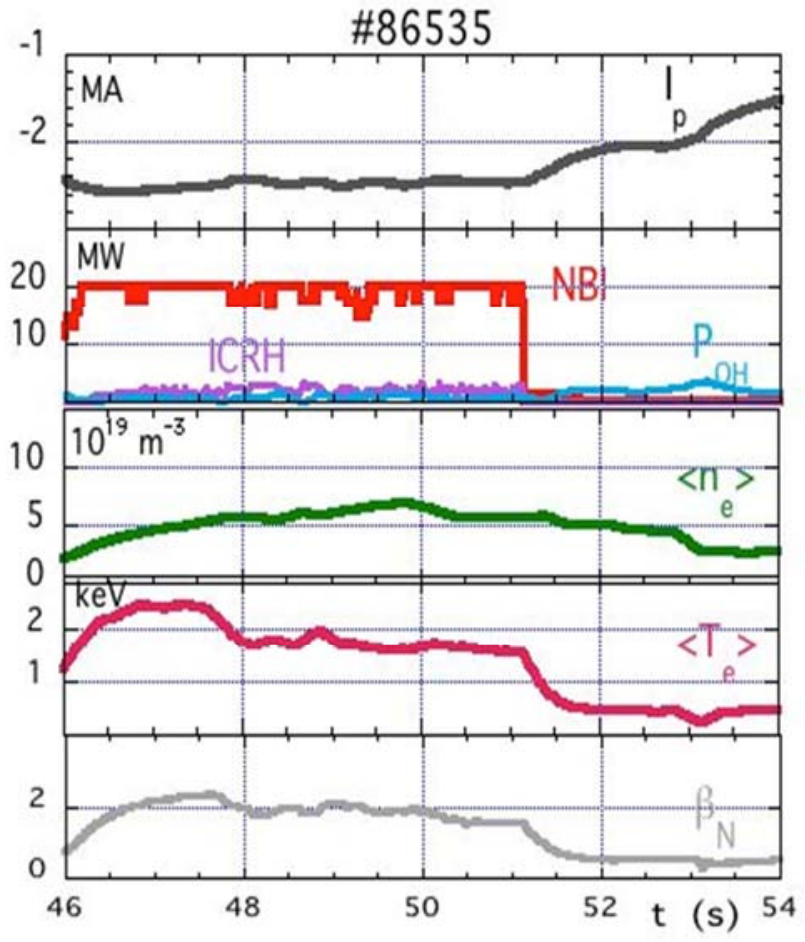

(b)

Figure 3. Time traces of main plasma parameters of JET discharges \#76791 (a) and \#86535 (b). From the top to the bottom we have: plasma current (MA), the additional heating source (NBI, $I C R H$, ohmic) in $M W$, the mean electron density $\left(10^{19} \mathrm{~m}^{3}\right)$, the mean electron temperature (keV), the normalized beta. 


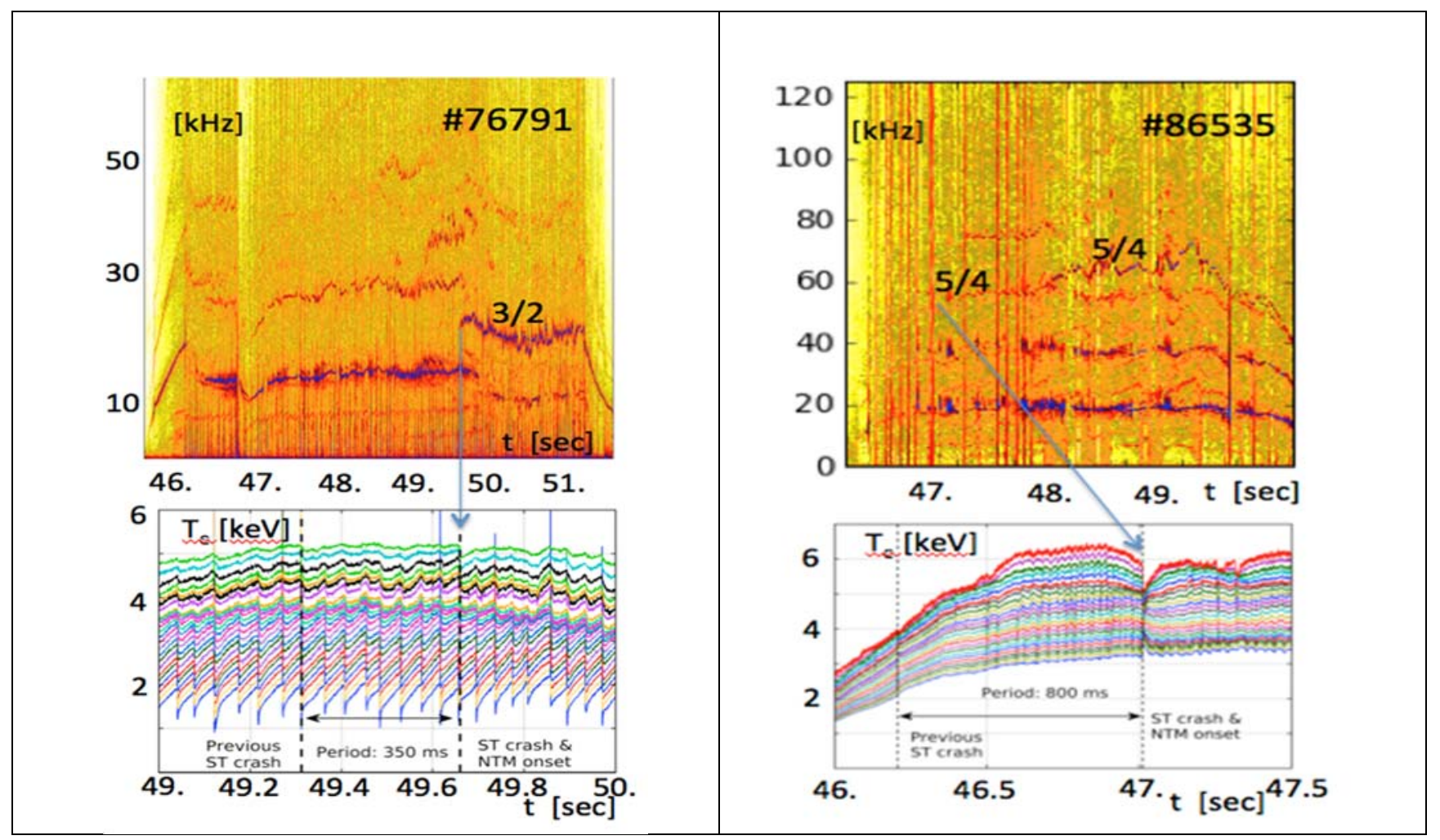

Figure 4. Observed NTM triggering (spectrograms on the top) after a sawtooth crash (bottom) for \#76791 (left) and \#86535 (right) shots. The spectrograms are provided by the Giovannozzi's code [33]. The bottom panel shows the experimental ECE radiation temperature from core channels for both shots during the last sawtooth period before the NTM onset. For shot \#76791 (left), the sawteeth crashes are almost simultaneous with ELM crashes. Several ELM crashes can be seen during the sawtooth period affecting mostly the lower temperature channels. 


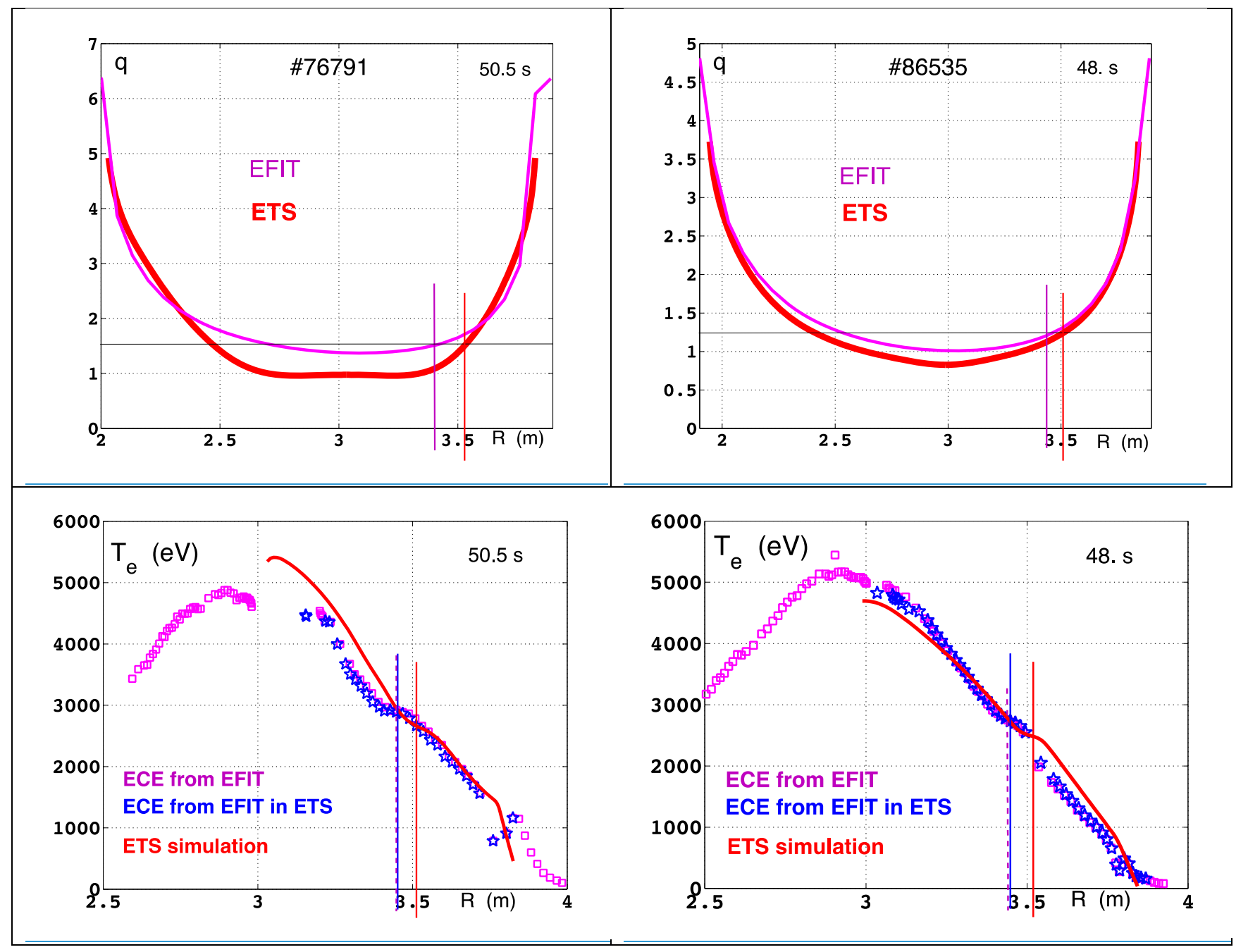

Figure 5. Comparison of the safety factor and electron temperature profiles vs major radius from EFIT and ETS reconstruction for the JET discharges \#76791 (left) and \#86535 (right). A difference between EFIT and ETS mode location (3/2 for \#76791 and 5/4 for \#86535) is found due to the uncertainties $(\leq 10 \%)$ of the equilibrium mapping (location of the magnetic axis) since EFIT has no adequate internal constraints. In the figures on the bottom the red continuous lines refer to the ETS simulation 


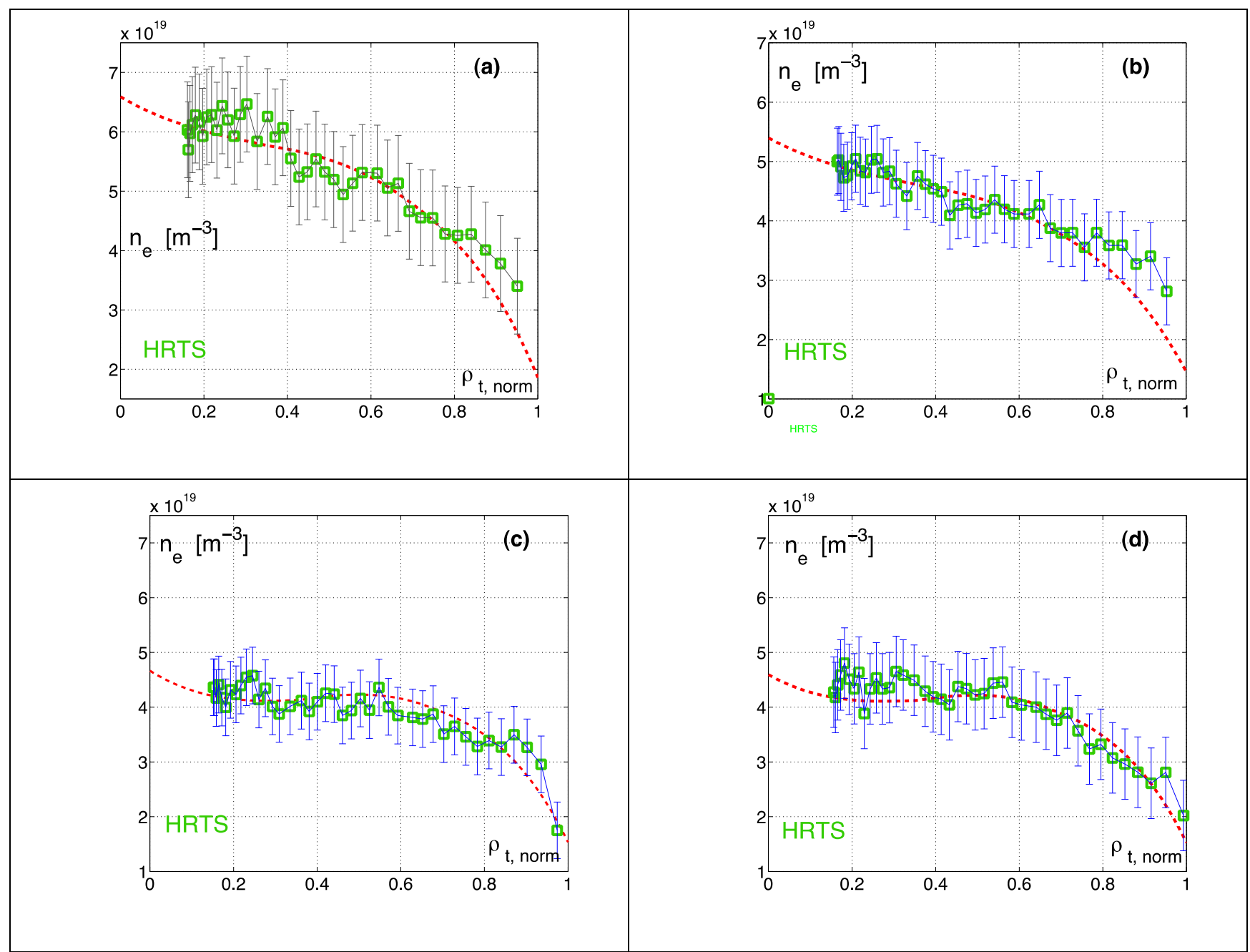

Figure 6. JET \#76791 - electron density profiles from HRTS diagnostic (squares) diagnostics at 48s before the $3 / 2$ onset (a) and $49.9 \mathrm{~s}$ (b), $50.5 \mathrm{~s}$ (c) and $50.7 \mathrm{~s}$ (d) after the mode triggering with corresponding errorbars and profiles prescribed and not recalculated by ETS without the presence of NTM (dashed lines). For the first 2 times (plots on the top) the profiles in ETS marginally fit the measurements at the edge, but they are in very good agreement with the last 2 times during the NTM phase (plots on the bottom) 


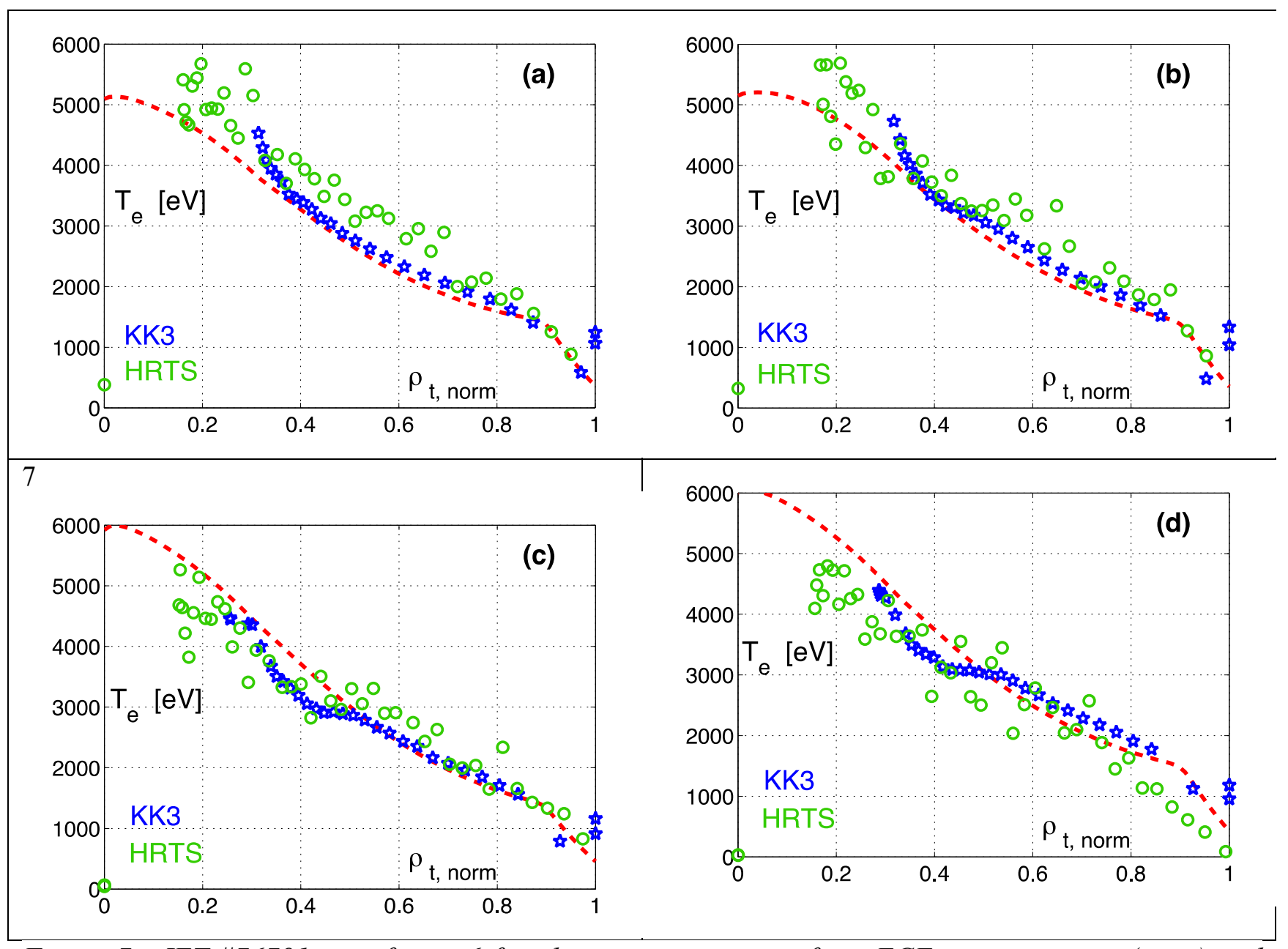

Figure 7. JET \#76791 - as figure 6 for electron temperature from ECE measurements (stars) and HRTS (open circles) diagnostics and recalculated by ETS in the second predictive phase without NTM (dashed lines). The plots are still considered at $48 s$ (a) before the mode onset and at 49.9s (b), $50.5 \mathrm{~s}(\mathrm{c})$ and $50.7 \mathrm{~s}$ (d) after the mode appearance) 


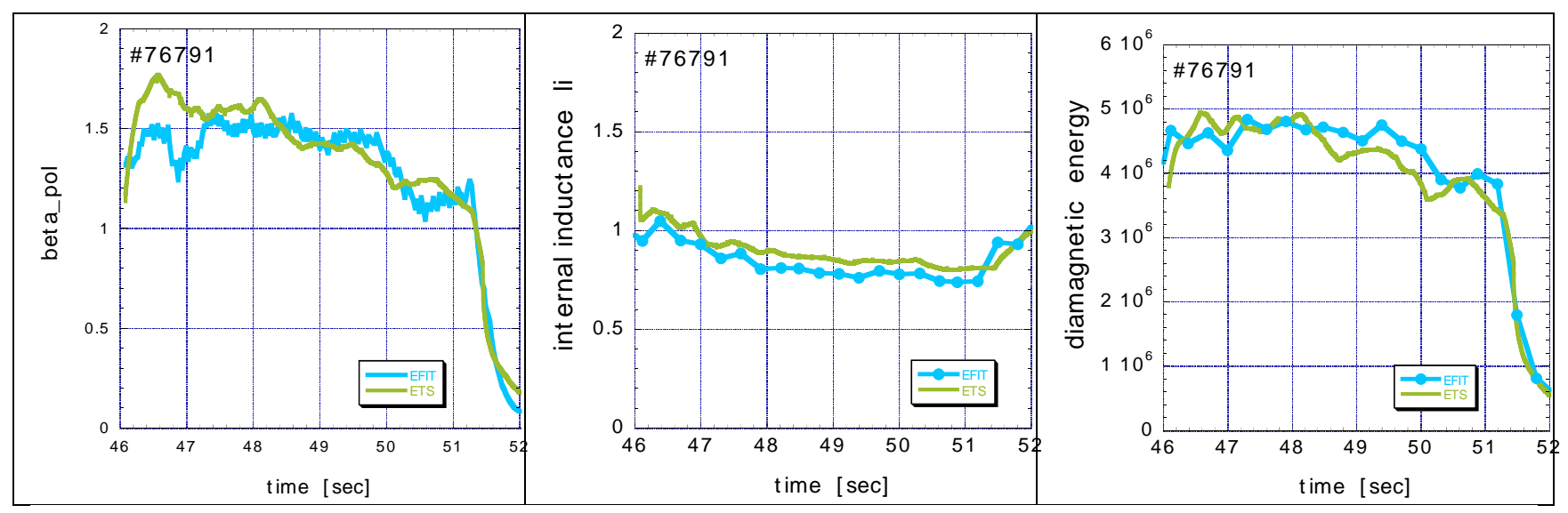

Figure 8. JET \#76791 - comparison of experiments (EFIT) and calculated quantities (ETS) of $\beta_{\text {pol }}$, internal inductance $\left(l_{i}\right)$ and diamagnetic energy $\left(W_{\text {dia }}\right)$ time traces.
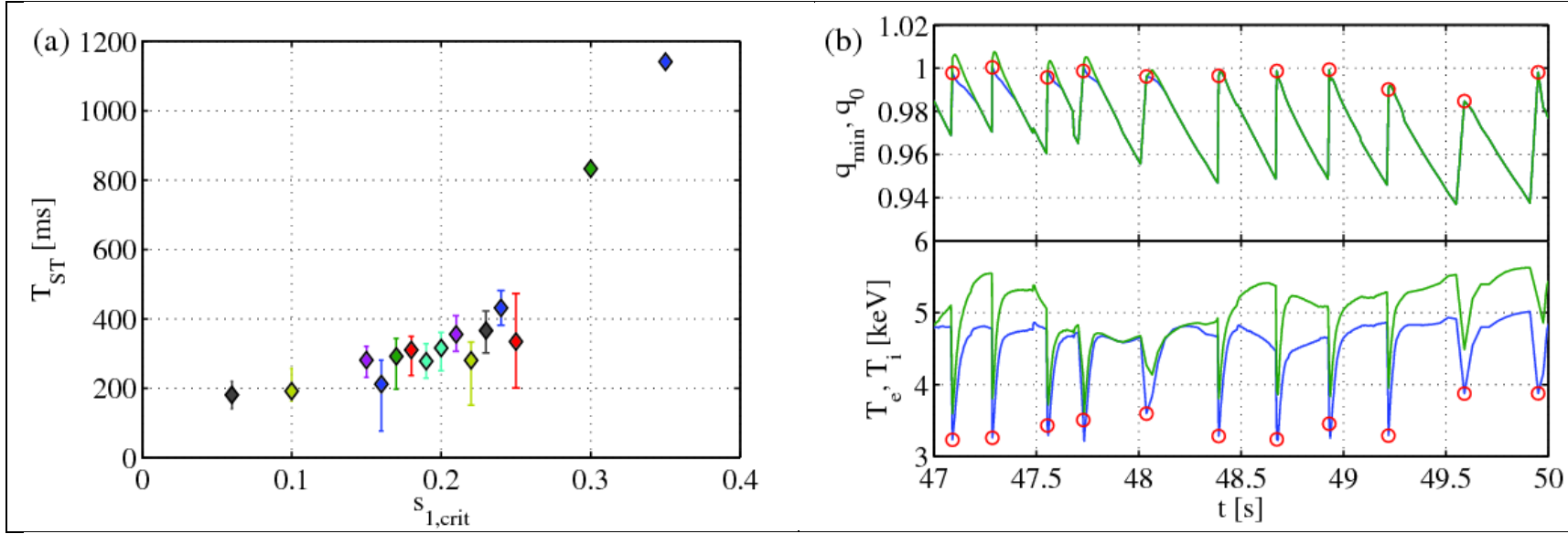

Figure 9. JET \#76791 - (a) Effect of changing the critical shear at the $q=1$ surface on the simulated sawtooth period. The experimental period $T_{S T}=350 \mathrm{~ms}$ is recovered for $s_{1, \text { crit }}=0.2$. (b) Evolution of the central values of $q, T_{e}, T_{i}$ for $c_{*}$ corresponding to $s_{1, \text { crit }}=0.2$. The period increases with time before the experimental time of the NTM onset. 


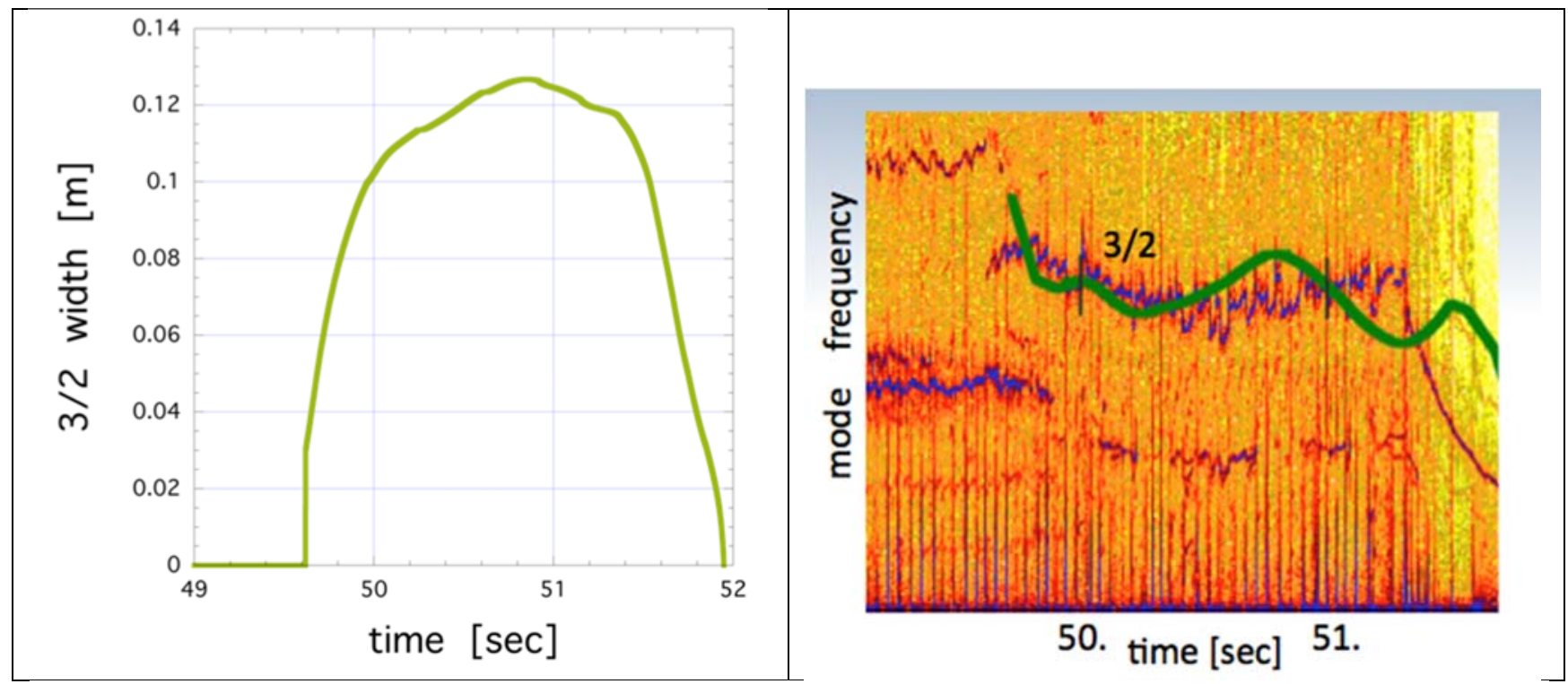

Figure 10. JET \#76791 - calculated size (left) and frequency (right) evolution of the 3/2 mode. The shape in the plasma frame of calculated frequency time trace (solid continuous green line) is overlapped on the experimental one in laboratory frame in the spectrogram. The viscous torque affects the change of the frequency slope as discussed in Sect. 2.3. The simulation is in nearly agreement with the experimental trace until about 50.3s and then the positive slope is obtained before the observed one. This is due perhaps to the fast change in sign of ( $\omega-\omega * i)$ (see Eq. (1.6)). Again the spectrogram is provided by the Giovannozzi's code [33] 


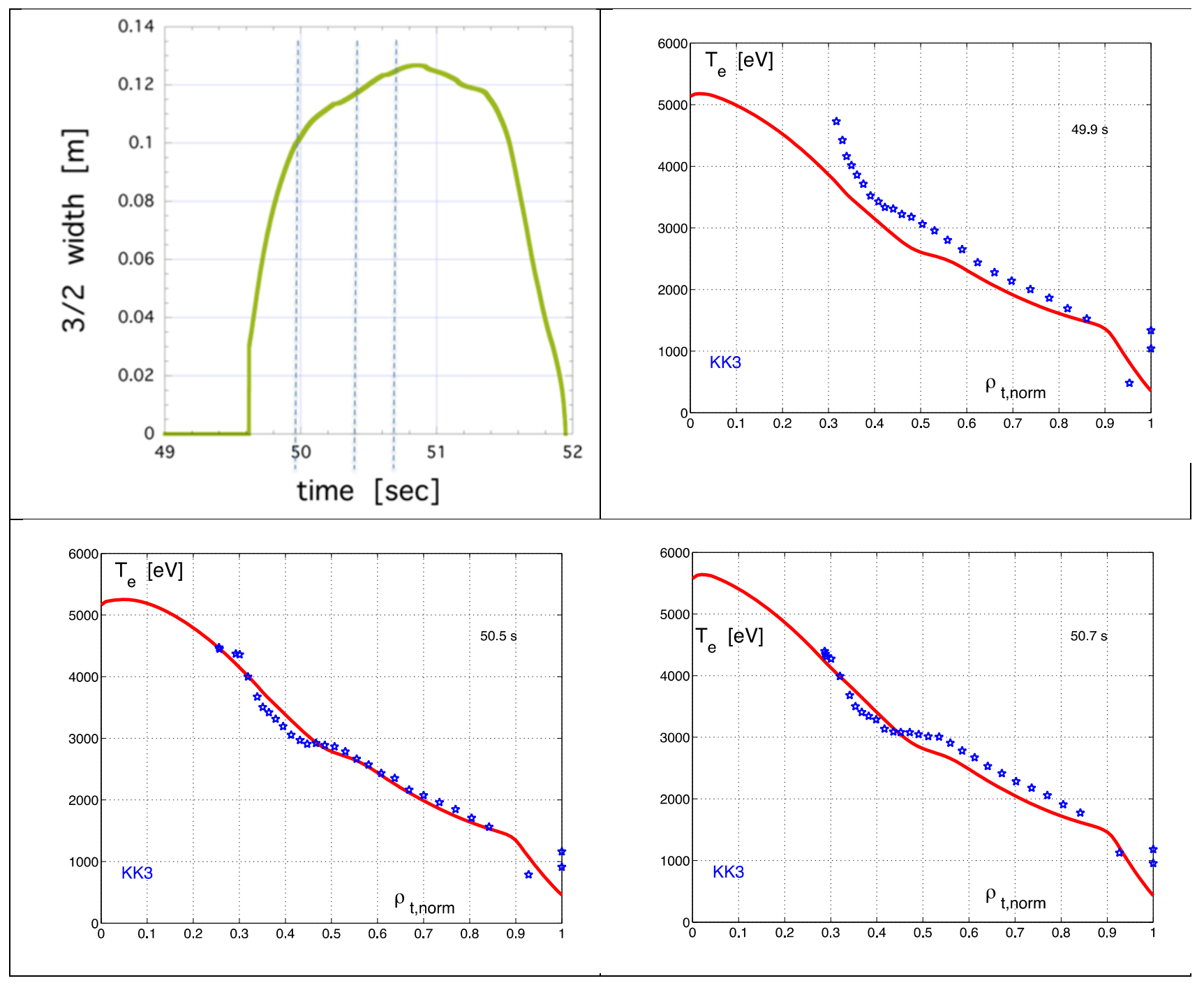

Figure 11. JET \#76791 - electron temperature at 49.9s (top right), 50.5s (bottom left) and 50.7s (bottom right) after the 3/2 mode appearance. The experimental (stars) from ECE measurements and the calculated (continuous line) mode size (profile flattening) are in fairly agreement. For the simulated traces Gaussian perturbations (at the mode location) of the electron transport coefficients have been considered, using $c_{w \chi}{ }=1$ and $c_{\chi}$ Te $=20$ (see Eq. (1.7)) 


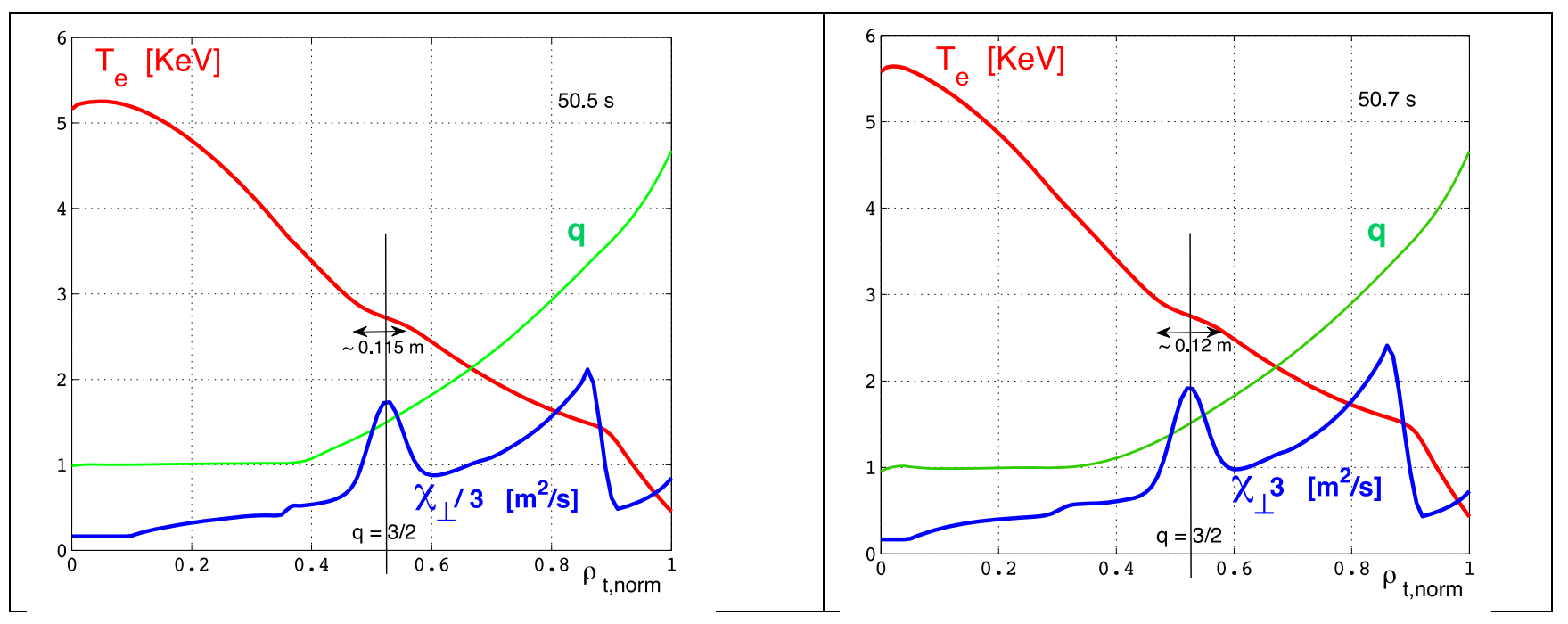

Figure 12. JET \#76791 - safety factor q, electron temperature $T_{e}$ and perpendicular diffusion coefficient $c_{\perp}$ profiles at $48 \mathrm{~s}$ before the 3/2 mode onset (left) and at $50.7 \mathrm{~s}$ after the 3/2 mode appearance (right). In this plot the flattening of the electron temperature profile due to the mode presence has been obtained modifying the $c_{\perp}$ coefficient by the same Gaussian function of figure 11 

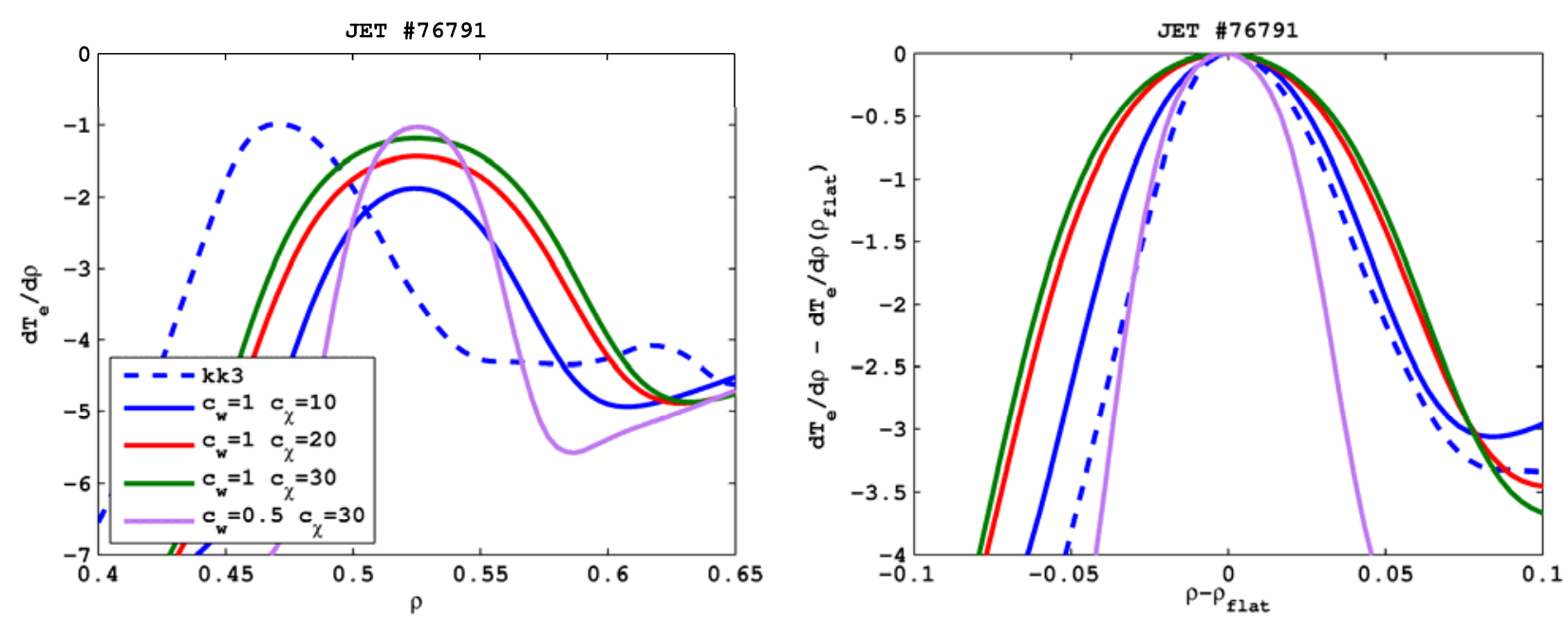

Figure 13. (left) $d T_{e} / d \rho$ versus $\rho$, the coefficient $c_{\chi}$ determines the "level" of flattening and therefore how close to zero is the "minimum" derivative. We see that 10 leads to a flattening similar to the measurements, while 20 or more give similar result. The coefficient $c_{w}$ determines the width of the main flattening which is better compared by shifting $d T_{e} / d \rho$ and $r$ around the rational surface of interest (right). We see that $c_{w}=0.5$ is too small and $c_{w}=1$ well reproduces the experimental data.
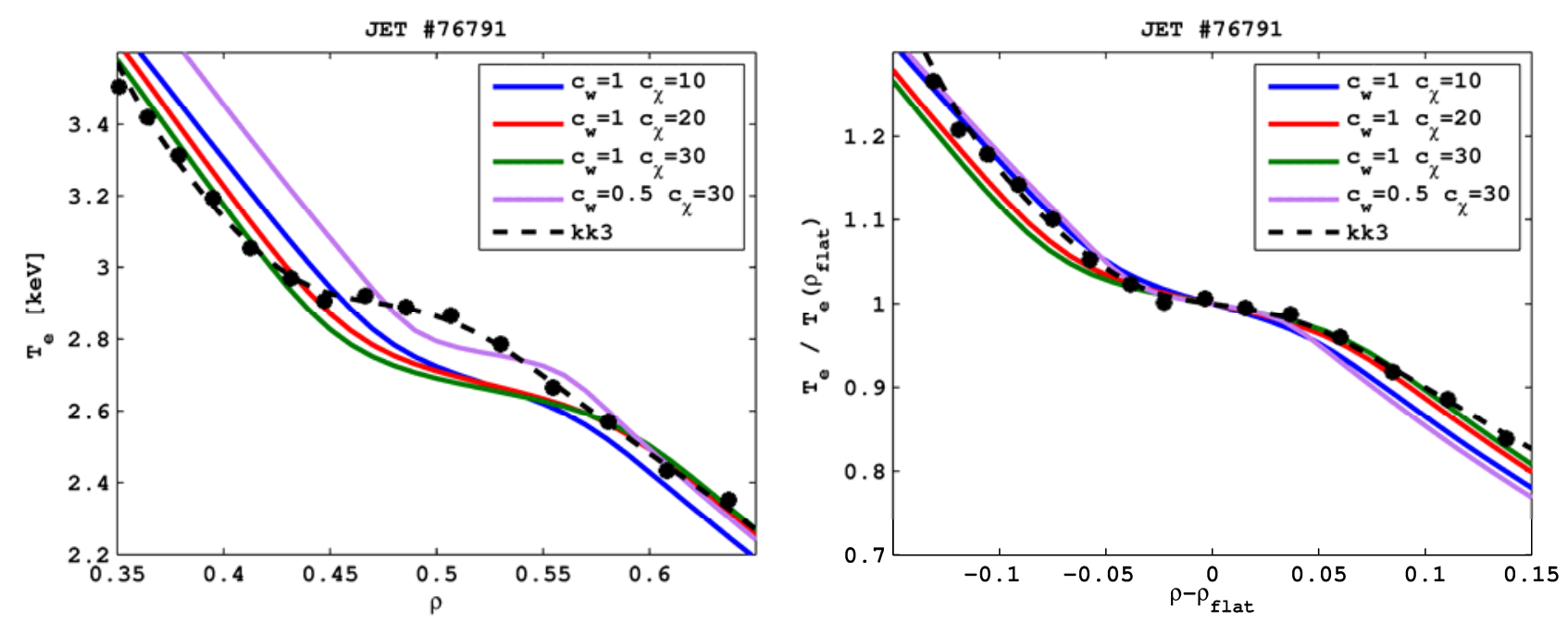

Figure 14. (left) $T_{e}$ versus $\rho$, and (right) $T_{e} / T_{e}$ ( $\left.\rho_{\text {flat }}\right)$ against $\left(\rho-\rho_{\text {flat }}\right)$ for the same values of $c_{w}$ and $c_{\chi}$ as in Fig. 13. The results confirm that the experimental observations with $c_{w}=1$ and $c_{\chi}=10$ are well recovered and that with $c_{\chi}, \geq 20$ profiles do not change too much. In the plot on the left the calculated profiles are shifted only because the $q=m / n$ surface is slightly different. 

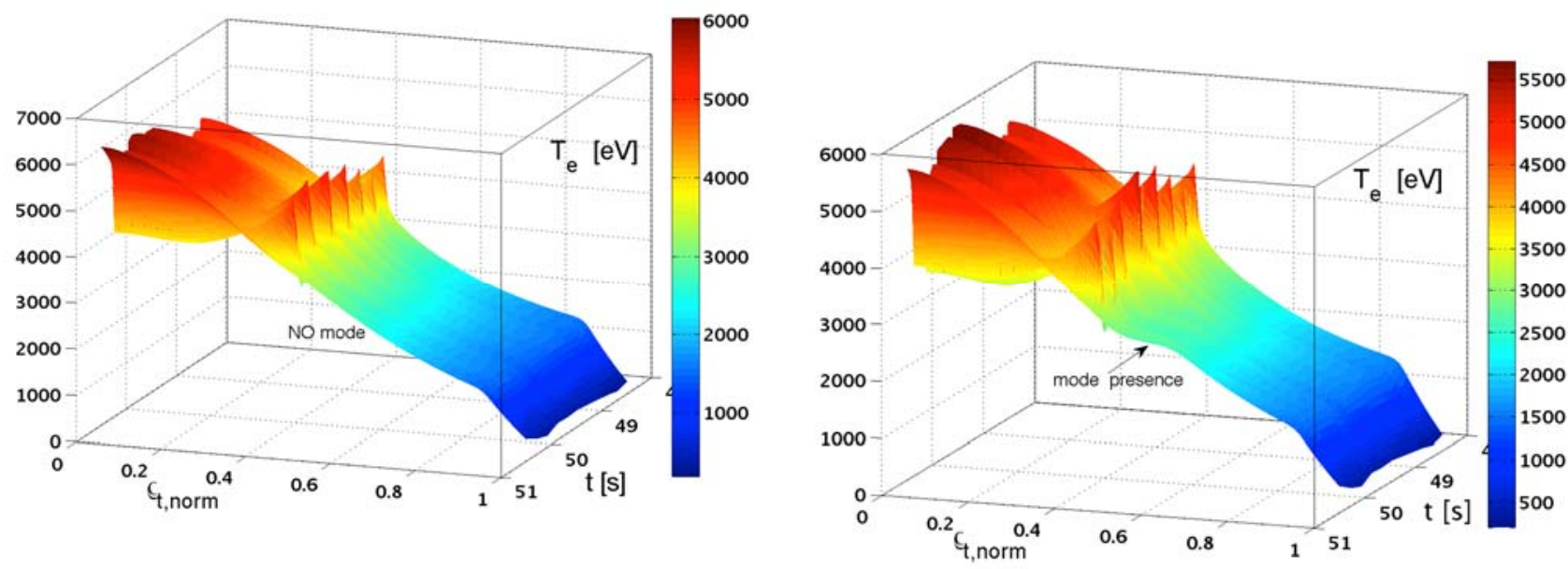

Figure 15. JET \#76791 - comparison of electron temperature contour plots calculated for the discharge \# 76791 without (left) and with (right) the modeling of the 3/2 mode. In the latter case, the modified shape of the iso-temperature lines in the experimental time interval of the observed $3 / 2$ NTM, are due to the changes of the electron and ion temperature transport coefficients.
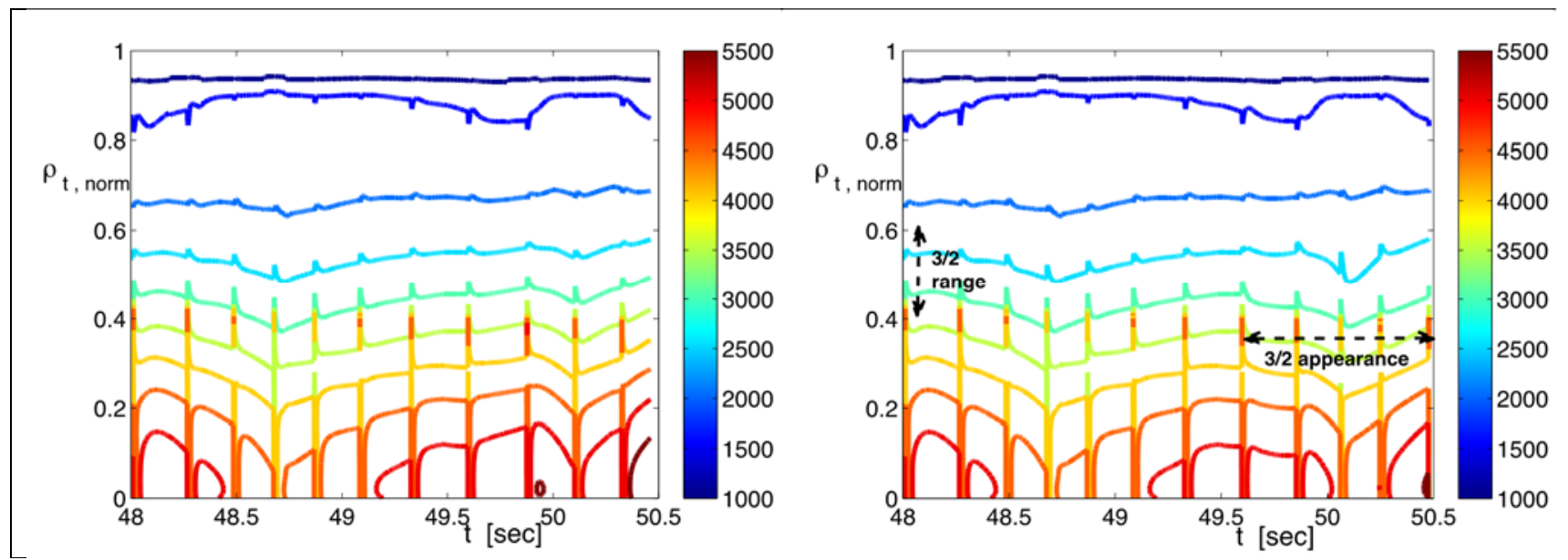

Figure 16. JET \#76791 - comparison of electron temperature contour plots calculated for the discharge \# 76791 without (left) and with (right) the modeling of the 3/2 mode. In the latter case, the modified shape of the iso-temperature lines in the experimental time interval of the observed $3 / 2$ NTM, are due to the changes of the electron and ion temperature transport coefficients. 


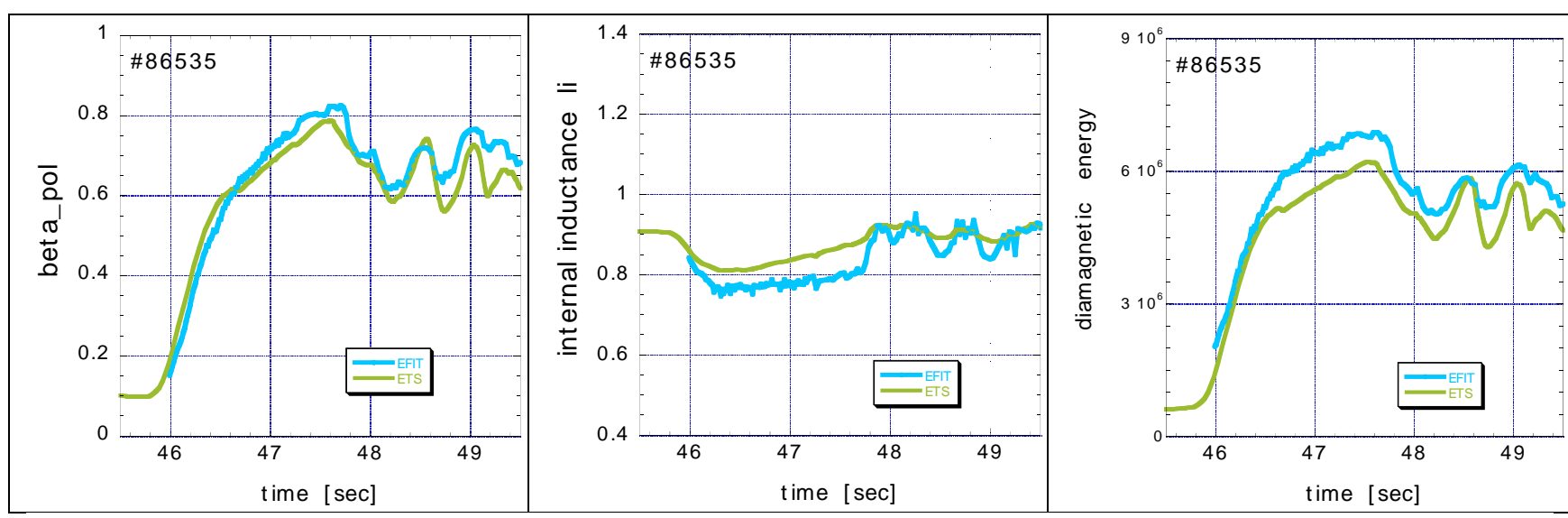

Figure 17. JET \#86535- comparison of evaluations from experiments (EFIT) and calculated quantities (ETS) of $\beta_{\text {pol }}$, internal inductance $\left(l_{i}\right)$ and diamagnetic energy ( $\left.W_{\text {dia }}\right)$ time traces for JET \#86535. 

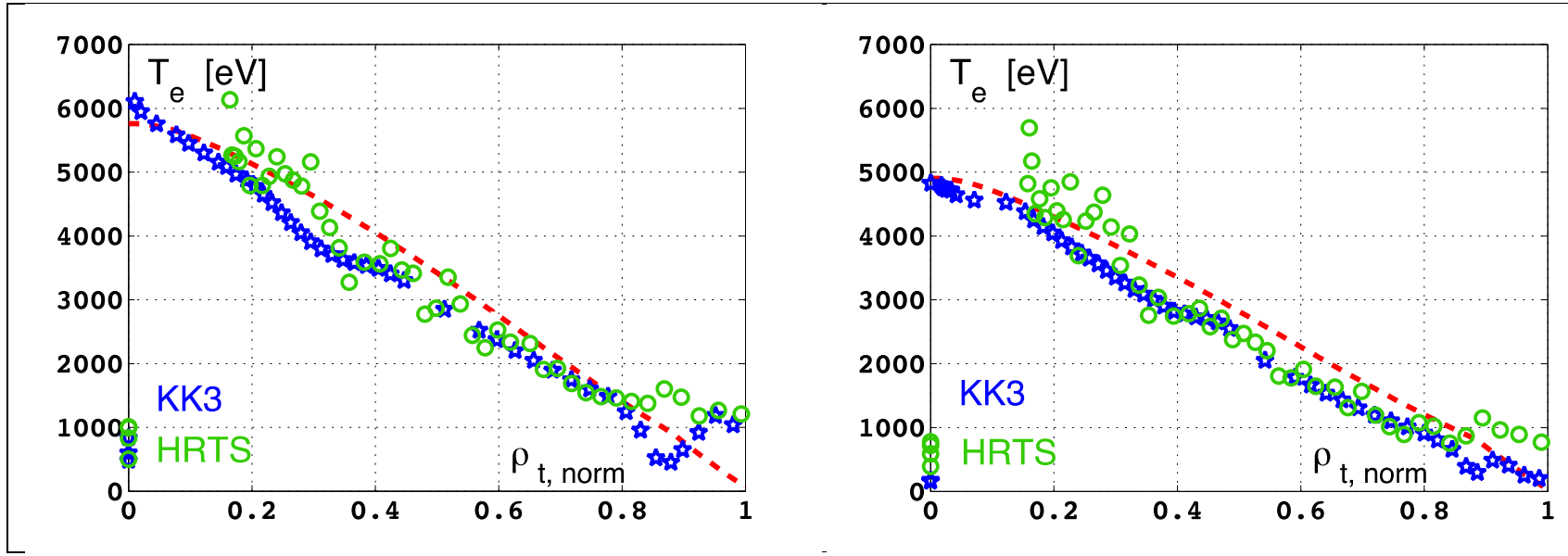

Figure 18. JET \#86535 - electron temperature from KK3 (stars) and HRTS (open circles) diagnostics at 47.6s (left) and 48.s (right) and profiles calculated in predictive phase without the presence of an NTM (dashed lines). The mode sizes evaluated from the temperature flattening are marked.
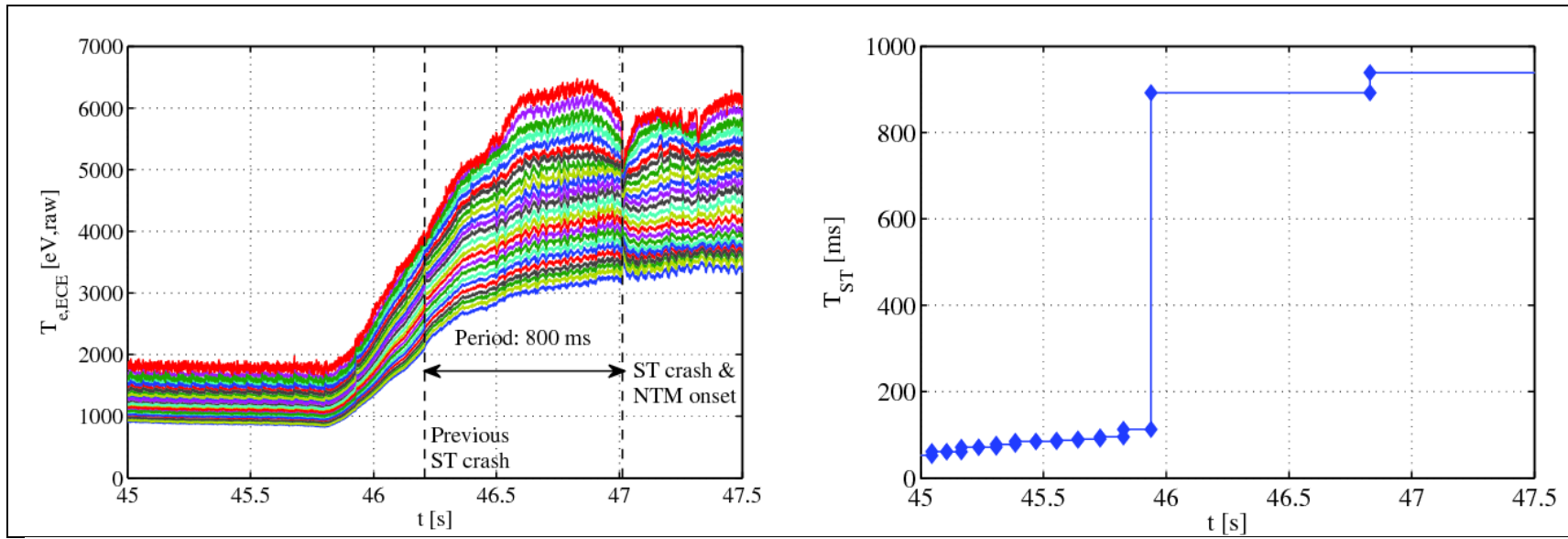

Figure 19. JET \#86535 - Raw electron temperature traces from ECE emission, highlighted are the long sawtooth crash leading to the NTM onset around $t=47.0 \mathrm{~s}$ (left). Evolution of the simulated sawtooth period showing a strong increase between the L-and H-mode phases (right). In both phases the simulated and experimental sawtooth period are in reasonable agreement. 


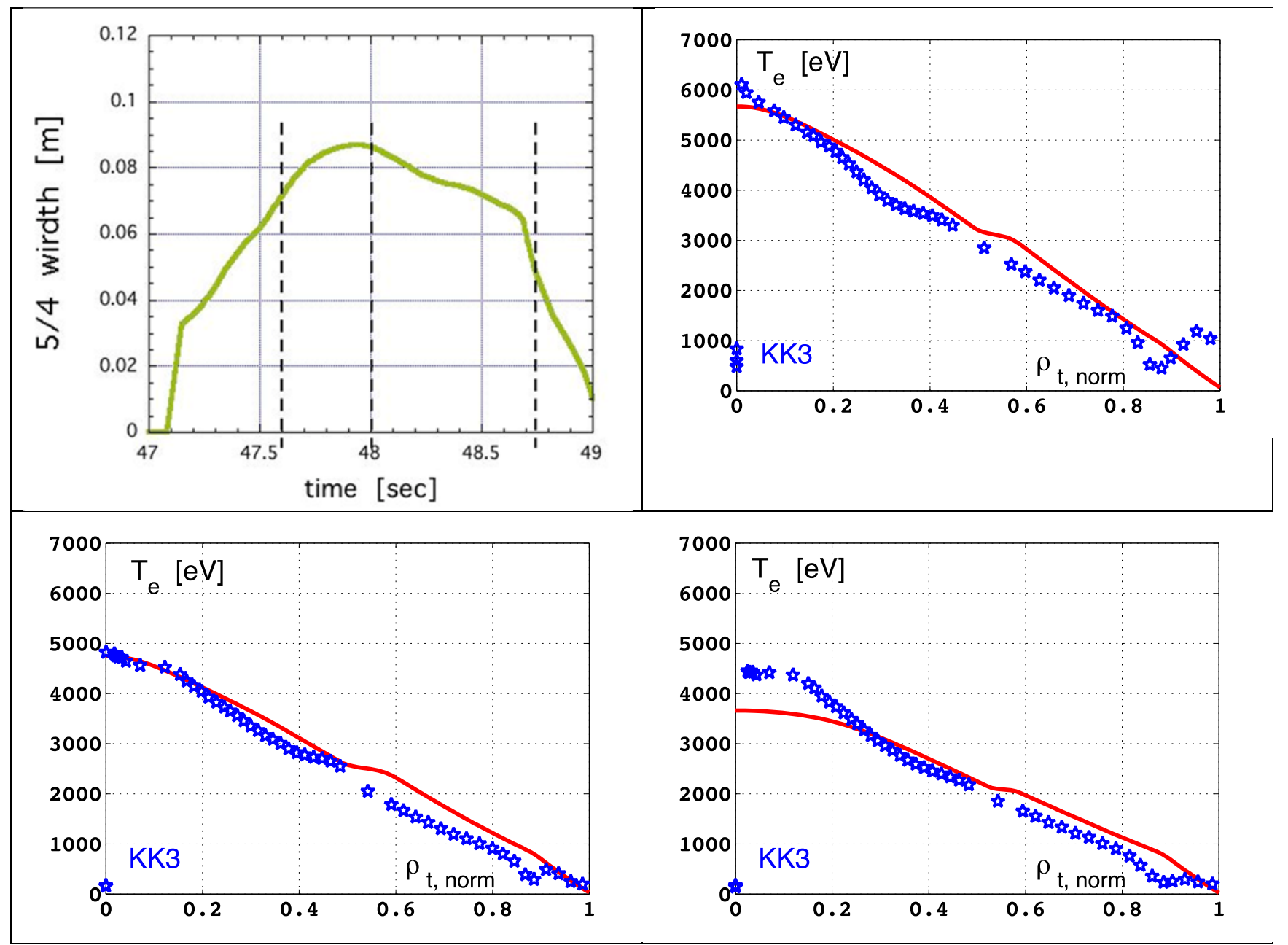

Figure 20. JET \#86535 - 5/4 mode size evolution (top left) and comparison of electron temperature profiles from ECE measurements (stars) and calculated ones (continuous lines) at 47.6s (top right), 48.s (bottom left) and 48.75s (bottom right) after the 5/4 mode appearance. The computed traces have been obtained considering Gaussian perturbations (at the mode location) of the electron transport coefficients with $c_{w \chi T e}=1$ and $c_{\chi, T e}=10$ (see Eq. (1.7)) 

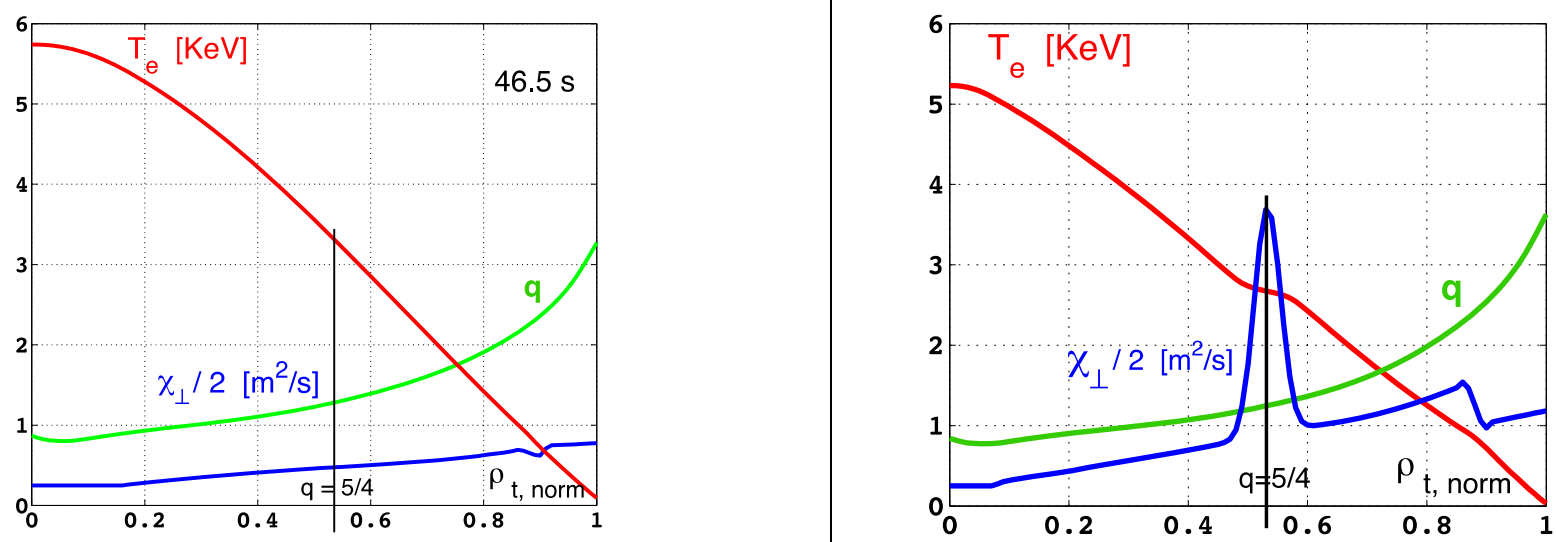

Figure 21. JET \#86535 - safety factor $q$, electron temperature $T_{e}$ and diffusion coefficient $c_{\perp}$ profiles at 46.5s before the 5/4 mode onset (left) and 48s after the 5/4 appearance (right). The flattening of the electron temperature profile at the mode location has been obtained modifying the $c_{\perp}$ coefficient with the same Gaussian function of figure 20
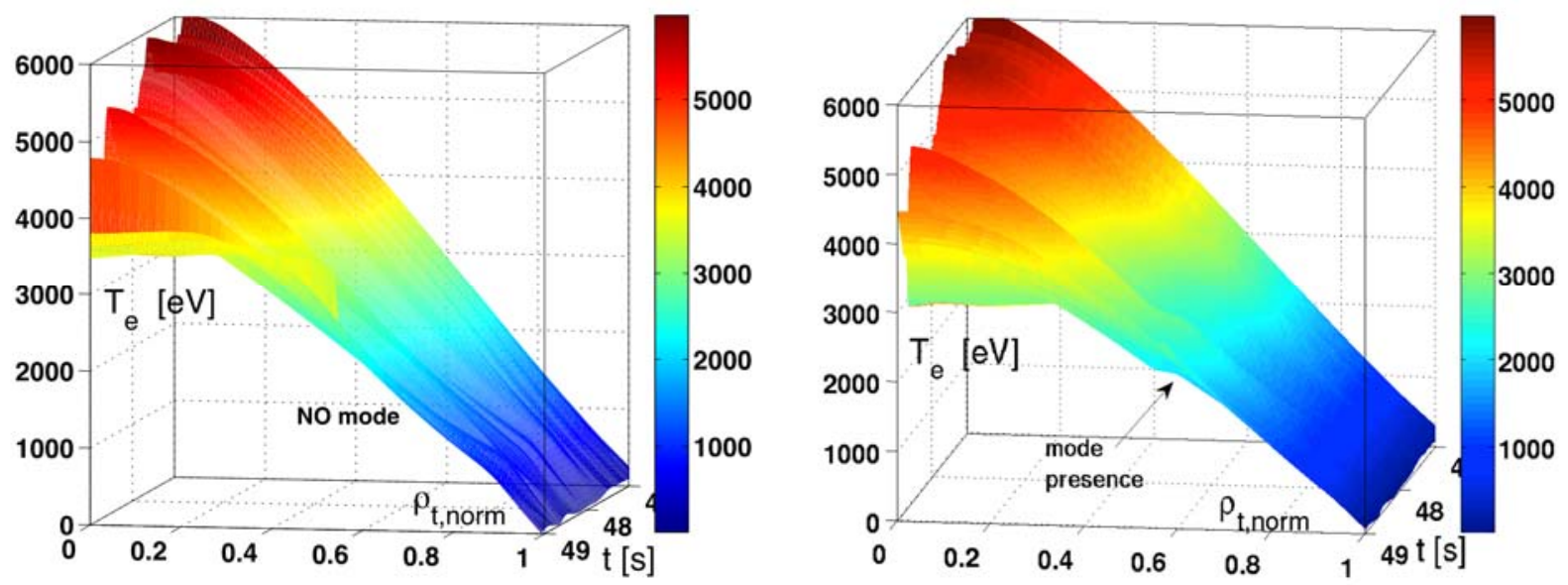

Figure 22: JET \#86535 - 3D electron temperature plots without (left) and with (right) the NTM modeling. 


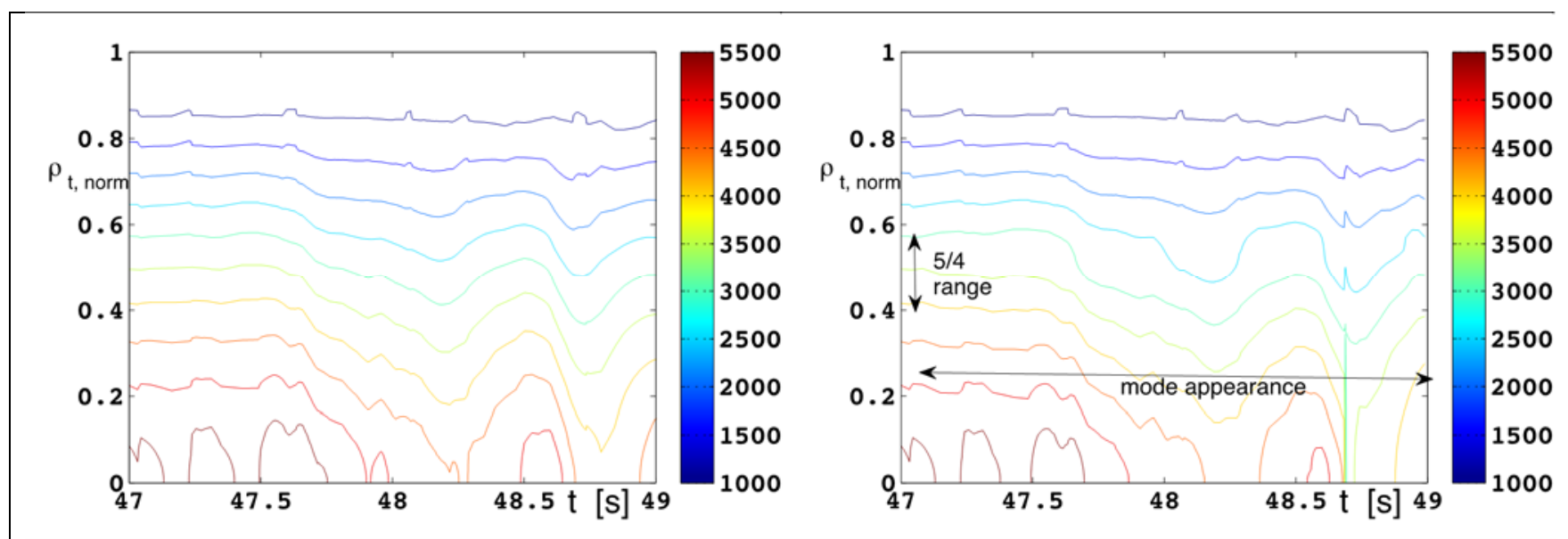

Figure 23. JET \#86535 - comparison of electron temperature contour plots calculated for the discharge \# 86535 without (left) and with (right) the modeling of the 5/4 mode. In the latter case, the modified shape of the iso-temperature lines in the experimental time interval of the observed 5/4 NTM, are due to the changes of the electron and ion temperature transport coefficients. 